\title{
An overconvergent 0.5 (half) Eichler-Shimura isomorphism
}

\author{
Fabrizio Andreatta \\ Adrian Iovita \\ Glenn Stevens
}

February 18, 2015

\section{Contents}

1 Introduction $r$

2 Faltings' topoi $\quad 4$

2.1 The geometric set-up. . . . . . . . . . . . . . . . . . . . 4

2.2 Faltings' topos . . . . . . . . . . . . . . . . . . . . . . . . . . . . . . . . . . . . . .

2.3 Sheaves on $\mathfrak{X}(w) \quad \ldots \ldots \ldots \ldots \ldots \ldots$

2.4 The localization functors . . . . . . . . . . . . . . . . 6

3 Locally Analytic Modular Symbols $\quad 7$

3.1 Accessible weights . . . . . . . . . . . . . . . . . . . . . 7

3.2 Analytic functions and distributions . . . . . . . . . . . . . . . 8

3.3 The geometric picture ............................. 9

4 Modular sheaves $r$

4.1 The sheaves $\Omega_{\mathfrak{X}}^{k}(w) \ldots \ldots \ldots \ldots \ldots \ldots$

4.2 The sheaves $\mathbb{D}_{k}$, and $\mathbb{D}_{k}^{m} \ldots \ldots \ldots \ldots \ldots \ldots \ldots$

4.3 The map $\operatorname{dlog}^{\vee}, k \ldots \ldots \ldots \ldots \ldots$

4.4 The cohomology of the sheaves $\omega_{\mathfrak{X}(w)}^{\dagger, k} \ldots \ldots \ldots \ldots \ldots \ldots$

5 The main result $r$

5.1 The cohomology of the quotients $\mathbb{D}_{k, K}^{m} / \mathbb{D}_{k, K}^{m+n} \ldots \ldots \ldots \ldots$

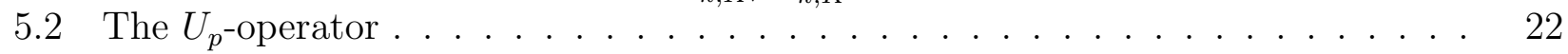

5.3 Slope decomposition . . . . . . . . . . . . . . . . . . . 24 


\section{Introduction}

In the recent preprint $[\mathrm{Sz}], \mathrm{P}$. Scholze proved, in much greater generality than quoted here, the following results. Let $N \geq 3$ be an integer, $p \geq 3$ a prime integer which does not divide $N$ and consider the tower

$$
\cdots \longrightarrow\left(Y_{N p^{r+1}} \subset X_{N p^{r+1}}\right) \longrightarrow\left(Y_{N p^{r}} \subset X_{N p^{r}}\right) \longrightarrow \ldots
$$

for $r \geq 0$, where $Y_{N p^{r}}$ denotes the affine modular curve over $\mathbb{C}_{p}$ with full level $N p^{r}$ structure and $X_{N p^{r}}$ its natural compactification, both seen as adic spaces over $\mathrm{Spa}\left(\mathbb{C}_{p}, \mathcal{O}_{\mathbb{C}_{p}}\right)$. The morphisms in the tower are the natural (forgetful) maps. Then we have:

- (Theorem IV.1.1 $[\mathrm{Sz}])$. There is an adic perfectoid space $X_{N p^{\infty}}$ over $\operatorname{Spa}\left(\mathbb{C}_{p}, \mathcal{O}_{\mathbb{C}_{p}}\right)$ such that

$$
X_{N p^{\infty}} \sim \lim _{\leftarrow, r} X_{N p^{r}}
$$

where $\sim$ is defined in definition 2.4.1 of $[\mathrm{SzW}]$.

If we denote by $\widetilde{\mathrm{H}}_{c, N}^{1}\left(\mathbb{Z} / p^{n} \mathbb{Z}\right)$ the compactly supported cohomology of the tower with $\mathbb{Z} / p^{n} \mathbb{Z}$ coefficients, i.e.,

$$
\widetilde{\mathrm{H}}_{c, N}^{1}\left(\mathbb{Z} / p^{n} \mathbb{Z}\right):=\lim _{\rightarrow, r} \mathrm{H}_{c}^{1}\left(Y_{N p^{r}}, \mathbb{Z} / p^{n} \mathbb{Z}\right),
$$

then we have

- (Theorem IV.2.1 of $[\mathrm{Sz}]$ ). There is a natural isomorphism of almost $\mathcal{O}_{\mathbb{C}_{p}}$-modules

$$
\text { (*) } \quad \widetilde{\mathrm{H}}_{c, N}^{1}\left(\mathbb{Z} / p^{n} \mathbb{Z}\right) \otimes\left(\mathcal{O}_{\mathbb{C}_{p}} / p^{n} \mathcal{O}_{\mathbb{C}_{p}}\right) \cong \mathrm{H}^{1}\left(X_{N p^{\infty}}, \mathfrak{I}_{N p^{\infty}}^{+} / p^{n} \mathfrak{I}_{N p^{\infty}}^{+}\right),
$$

where $\mathfrak{I}_{N p^{\infty}} \subset \mathcal{O}_{X_{N p^{\infty}}}$ is the ideal sheaf of the boundary of $X_{N p^{\infty}}$ and $\mathfrak{I}_{N p^{\infty}}^{+}:=\mathfrak{I}_{N p^{\infty}} \cap \mathcal{O}_{X_{N p^{\infty}}}^{+}$.

One might want to think about the almost isomorphism $(*)$ above as a 0.5, i.e., a half, of a p-adic (or maybe perfectoid) Eichler-Shimura isomorphism as the left hand side is compactly supported completed, étale cohomology of the tower of modular curves and the right hand side is interpreted in $[\mathrm{Sz}]$ as the module of cuspidal $p$-adic modular forms.

In this article we prove yet another 0.5 Eichler-Shimura isomorphism, which arises in the context of overconvergent modular symbols and which is perhaps the missing half of $(*)$.

Let us recall that in the context of overconvergent modular symbols there are two notions corresponding to completed cohomology that we will now review. We now start using the notations used in the rest of this article: let $X(N, p) \longrightarrow X$ denote the pair consisting of the modular curve of level $\Gamma:=\Gamma_{1}(N) \cap \Gamma_{0}(p)$, respectively $\Gamma_{1}(N)$, over $\mathbb{Q}_{p}$ seen as a rigid analytic curves where the map is forget the level $p$-structure, let $0 \leq w \leq p /(p+1)$ be a rational number and let $K$ be a finite extension of $\mathbb{Q}_{p}$ such that there is an element $p^{w}$ of $K$ with $v\left(p^{w}\right)=w$. We denote by $X(w) \subset X$, respectively $X(w) \subset X(N, p)$ the strict neighborhood of the ordinary locus, respectively the strict neighborhood of the connected component of the ordinary locus containing the cusp $\infty$ in $X$ and $X(N, p)$ of width $w$ (see section 2.1 for more details). We let $\mathcal{X}(w)$ be the natural formal model over $\mathcal{O}_{K}$ of $X(w)$.

Let us fix an accessible weight $k$, i.e., a continuous homomorphism $k: \mathbb{Z}_{p}^{\times} \longrightarrow K^{\times}$which is analytic when restricted to $1+p \mathbb{Z}_{p}$ and let $D_{k}$ denote the module of $K$-valued analytic distributions on $T_{0}:=\mathbb{Z}_{p}^{\times} \times \mathbb{Z}_{p}$ which are homogeneous of weight $k$ for the natural action of $\mathbb{Z}_{p}^{\times}$ on $T_{0}$ (see section 3.2 for more details.)

The first analogue of the completed cohomology (associated to the tower of modular curves of levels $\left.\Gamma_{1}\left(N p^{r}\right), r \geq 0\right)$ is the Hecke module of overconvergent modular symbols of weight $k$, $\mathrm{H}^{1}\left(\Gamma, D_{k}\right)$. 
As $D_{k}$ can be seen as a continuous representation of the Kummer-étale geometric fundamental group of $X(N, p)$ (for a certain base point), associated to $D_{k}$ there is a continuous Kummerétale sheaf $\mathcal{D}_{k}$ on $X(N, p)$ such that we have $\mathrm{H}^{1}\left(\Gamma, D_{k}\right) \cong \mathrm{H}^{1}\left(X(N, p) \frac{\mathrm{ket}}{K}, \mathcal{D}_{k}\right)$. In particular $\mathrm{H}^{1}\left(\Gamma, D_{k}\right)$ has a natural action of the absolute Galois group $G_{K}$ of $K$.

Next, we can restrict the sheaf $\mathcal{D}_{k}$ to the Kummer-étale site of $X(w)$ and then consider it as a sheaf on Faltings' site $\mathfrak{X}(w)$ associated to the pair $(\mathcal{X}(w), X(w))$. Let also $\widehat{\mathcal{O}}_{\mathfrak{X}(w)}$ denote the continuous structure sheaf of $\mathfrak{X}(w)$. The second analogue of completed cohomology, for the tower of rigid curves $\left(X_{1}\left(N p^{r}\right)(w)\right)_{r \geq 0}$, would be the group $\mathrm{H}^{1}\left(\mathfrak{X}(w), \mathcal{D}_{k} \hat{\otimes} \widehat{\mathcal{O}}_{\mathfrak{X}(w)}\right)$. Let us recall that there is a $G_{K}$ and Hecke equivariant mysterious map (restriction) connecting the two "completed cohomology analogues" (see [AIS2]):

$$
\mathrm{H}^{1}\left(\Gamma, D_{k}\right) \otimes_{K} \mathbb{C}_{p} \longrightarrow \mathrm{H}^{1}\left(\mathfrak{X}(w), \mathcal{D}_{k} \hat{\otimes} \widehat{\mathcal{O}}_{\mathfrak{X}(w)}\right) .
$$

In [AIS2] we have proved a full but imperfect Eichler-Shimura isomorphism theorem for $\mathrm{H}^{1}\left(\Gamma, D_{k}\right)$ as follows: for a slope $h \geq 0$, there is a discrete set of bad weights $Z_{h}$ such that if $k$ is an accessible weight with $h \notin Z_{h}$, we have a $G_{K}$ and Hecke equivariant isomorphism

$$
\mathrm{H}^{1}\left(\Gamma, D_{k}(1)\right)^{(h)} \otimes_{K} \mathbb{C}_{p} \cong \mathrm{H}^{0}\left(X(w), \omega_{w}^{\dagger, k+2}\right)^{(h)} \otimes_{K} \mathbb{C}_{p} \oplus S_{k}^{(h)} \otimes_{K} \mathbb{C}_{p}\left(\chi^{k-1}\right),
$$

where the exponent $(h)$ denotes the factor of slope $\leq h$ of each of the $U_{p}$-modules above, $\omega_{w}^{\dagger, k+2}$ is the modular sheaf on $X(w)$ of weight $k+2$ and $S_{k}$ is a Hecke module on a Banach space on which $U_{p}$ is compact.

The goal of the present article is to prove an overconvergent 0.5 Eichler-Shimura isomorphism for $\mathrm{H}^{1}\left(\mathfrak{X}(w), \mathcal{D}_{k} \hat{\otimes} \widehat{\mathcal{O}}_{\mathfrak{X}(w)}\right)$. Let $k$ be an accessible weight and $h \geq 0$ a slope. We recall that there is a $G_{K}$ and Hecke equivariant map defined in [AIS2] and reviewed in section 5 of this article

$$
\mathfrak{R}_{k}: \mathrm{H}^{1}\left(\mathfrak{X}(w), \mathcal{D}_{k} \hat{\otimes} \widehat{\mathcal{O}}_{\mathfrak{X}(w)}(1)\right) \longrightarrow \mathrm{H}^{0}\left(X(w), \omega_{w}^{\dagger, k+2}\right) \otimes_{K} \mathbb{C}_{p} .
$$

Then we prove, see Theorem 5.1

Theorem 1.1. We have

a) The $\mathbb{C}_{p}$-vector space $\mathrm{H}^{1}\left(\mathfrak{X}(w), \mathcal{D}_{k} \hat{\otimes} \widehat{\mathcal{O}}_{\mathfrak{X}(w)}(1)\right)$ has a natural slope $h$-decomposition, and

b) $\mathfrak{R}_{k}$ induces an isomorphism

$$
\mathfrak{R}_{k}^{(h)}: \mathrm{H}^{1}\left(\mathfrak{X}(w), \mathcal{D}_{k} \hat{\otimes} \widehat{\mathcal{O}}_{\mathfrak{X}(w)}(1)\right)^{(h)} \cong \mathrm{H}^{0}\left(X(w), \omega_{w}^{\dagger, k+2}\right)^{(h)} \otimes_{K} \mathbb{C}_{p} .
$$

The proof of this theorem is based on the following facts:

1) The sheaf $\mathcal{D}_{k} \hat{\otimes} \widehat{\mathcal{O}}_{\mathfrak{X}(w)}$, contrary to $\mathcal{D}_{k}$, has a decreasing, separated and exhausting filtration by sub-sheaves, the cohomology of whose graded quotients can be calculated in terms of overconvergent modular forms.

2) One can estimate the $p$-adic valuation of the image of $U_{p}$ on the cohomology of the filtration of $\mathcal{D}_{k} \hat{\otimes} \widehat{\mathcal{O}}_{\mathfrak{X}(w)}(1)$.

We think that the fact that the cohomology group $\mathrm{H}^{1}\left(\mathfrak{X}(w), \mathcal{D}_{k} \hat{\otimes} \widehat{\mathcal{O}}_{\mathfrak{X}(w)}(1)\right)$ has a slope $h$ decomposition is surprising as we know little of its topological properties. Let us recall that slope decompositions have been proved for projective Banach modules over Banach algebras and for modules of overconvergent modular symbols by first decomposing the perfect complexes computing the modular symbols. 
Such complexes are not available for Faltings' cohomology and therefore we think this result is interesting in itself. Moreover, we believe that these ideas could be used to define slope decompositions for other continuous cohomology groups of ind-continuous sheaves.

Notations In what follows we will denote by caligraphic letters $\mathcal{X}, \mathcal{Y}, \mathcal{Z}, \ldots \log$ formal schemes over $\mathcal{O}_{K}$ and by $\underline{\mathcal{X}}, \mathcal{Y}, \underline{\mathcal{Z}}$ respectively the formal schemes underlying $\mathcal{X}$ respectively $\mathcal{Y}$, respectively $\mathcal{Z}$. We will denote by $X, Y, Z, \ldots$ respectively the $\log$ rigid analytic generic fibers of $\mathcal{X}$, $\mathcal{Y}, \mathcal{Z}, \ldots$ and by $\underline{X}, \underline{Y}, \underline{Z}, \ldots$ respectively the underlying rigid spaces.

\section{Faltings' topoi}

\subsection{The geometric set-up.}

In this section we will recall the geometric set-up from [AIS2]. Let $p \geq 3$ be a prime integer, $K$ a complete discrete valuation field of characteristic 0 , ring of integers $\mathcal{O}_{K}$, maximal ideal $\underline{m}_{K}$ of $\mathcal{O}_{K}$ and perfect residue field $\mathbb{F}$ of characteristic $p$. Let $N \geq 3$ be a positive integer not divisible by $p$. We fix once for all an algebraic closure $\bar{K}$ of $K$ and an embedding $\overline{\mathbb{Q}} \hookrightarrow \bar{K}$, where $\overline{\mathbb{Q}}$ is the algebraic closure of $\mathbb{Q}$ in $\mathbb{C}$. We denote by $\mathbb{C}_{p}$ the completion of $\bar{K}$ and by $G_{K}$ the Galois group of $\bar{K}$ over $K$. We denote by $v$ the valuation on $\mathbb{C}_{p}$, normalized such that $v(p)=1$.

Let $w \in \mathbb{Q}$ be such that $0 \leq w \leq p /(p+1)$ and let us suppose that there is an element (which will be denoted $p^{v}$ ) in $K$ whose valuation is $v:=w /(p-1)$. We fix an integer $r \geq 1$ and we suppose that $w<2 /\left(p^{r}-1\right)$ if $p>3$ and $w<1 / 3^{r}$ if $p=3$.

We consider the following tower of rigid analytic modular curves over $K$, with log structures defined by the divisors of cusps:

$$
X_{1}\left(N p^{r}\right) \longrightarrow X\left(N, p^{r}\right) \longrightarrow X_{1}(N),
$$

where $X_{1}\left(N p^{r}\right)$, respectively $X_{1}(N)$ classify generalized elliptic curves with $\Gamma_{1}\left(N p^{r}\right)$ (respectively $\left.\Gamma_{1}(N)\right)$ level structure, while $X\left(N, p^{r}\right)$ classifies generalized elliptic curves with $\Gamma_{1}(N) \cap \Gamma_{0}\left(p^{r}\right)$ level structure. The morphism $X\left(N, p^{r}\right) \longrightarrow X_{1}(N)$ is the one which forgets the $\Gamma_{0}\left(p^{r}\right)$-level structure.

We denote by $\mathrm{Ha}$ a lift of the Hasse invariant (for example $\mathrm{Ha}=E_{p-1}$, the normalized Eisenstein series of level 1 and weight $p-1$, if $p>3$ ) which we view as a modular form on $X_{1}(N)$. We define the rigid analytic space

$$
X(w):=\left\{x \in X_{1}(N) \quad|\quad| \mathrm{Ha}(x) \mid \geq p^{-w}\right\} \subset X_{1}(N),
$$

and remark that the morphism $X\left(N, p^{r}\right) \longrightarrow X_{1}(N)$ has a canonical section over $X(w)$, defined via the theory of the canonical subgroup, whose image we also denote by $X(w))$. We define $X\left(p^{r}\right)(w):=X_{1}\left(N p^{r}\right) \times_{X_{1}\left(N, p^{r}\right)} X(w)$ and view $X(w)$ (respectively $X\left(p^{r}\right)(w)$ ) as a connected affinoid subdomain of $X_{1}(N)$ and via the above mentioned section of $X\left(N, p^{r}\right)$ (respectively of $\left.X_{1}\left(N p^{r}\right)\right)$. The log structures are defined by the divisors of the cusps in $X\left(p^{r}\right)(w)$.

We denote by $\mathcal{X}_{1}(N), \mathcal{X}\left(N, p^{r}\right)$ and $\mathcal{X}_{1}\left(N p^{r}\right)$ the $p$-adic formal schemes over $\mathcal{O}_{K}$ obtained by completing the proper schemes over $\mathcal{O}_{K}$ classifying generalized elliptic curves with $\Gamma_{1}(N)$, respectively $\Gamma_{1}(N) \cap \Gamma_{0}\left(p^{r}\right)$, respectively $\Gamma_{1}\left(N p^{r}\right)$-level structures along, respectively, their special fibers. Let $\mathcal{X}(w)$ denote the open formal sub-scheme of the formal blow-up of $\mathcal{X}_{1}(N)$ defined by the ideal sheaf of $\mathcal{O}_{\mathcal{X}_{1}(N)}$ generated by the sections $p^{w}$ and $\mathrm{Ha}\left(\mathcal{E} / \mathcal{X}_{1}(N), \omega\right)$ which is the complement of the section at $\infty$ of the exceptional divisor of the blowing-up. Here $\mathcal{E} \longrightarrow \mathcal{X}_{1}(N)$ is the universal generalized elliptic curve and $\omega$ is a global invariant 1-differential form of $\mathcal{E}$ over 
$\mathcal{X}_{1}(N)$. Finally we let $\mathcal{X}\left(p^{r}\right)(w)$ denote the normalization of $\mathcal{X}(w)$ in $X_{1}(N p)(w)$ (see $\S 2.2$ of [AIS2] for more details). These formal schemes have natural log structures as follows: for $\mathcal{X}(w)$ it is defined by the divisors of the special fibers plus the divisors of the cusps, respectively (see [AIS2] §2.2.). If we denote by $\mathcal{S}$ the formal scheme $\operatorname{Spf}\left(\mathcal{O}_{K}\right)$ with $\log$ structure given by the closed point, then the natural morphism of log formal schemes $\mathcal{X}(w) \longrightarrow \mathcal{S}$ is $\log$ smooth.

The log structures on the formal schemes $\mathcal{X}\left(p^{r}\right)(w), r \geq 1$ are the inverse images of the $\log$ structure on $\mathcal{X}(w)$ defined by the morphisms of formal schemes $\mathcal{X}\left(p^{r}\right)(w) \longrightarrow \mathcal{X}(w)$.

Let us remark that we have constructed a natural commutative diagram of log formal schemes, log rigid analytic spaces and morphisms which is our basic geometric setup:

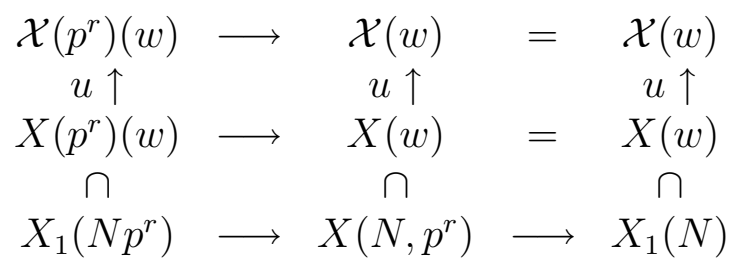

In the above diagram $u$ denotes the various specialization (or reduction) morphisms.

\section{$2.2 \quad$ Faltings' topos}

Our main reference for the constructions in this section is [AI] $§ 2.2$ and [AIS2] $§ 2.3$.

We will define Faltings' site, denoted $\mathfrak{X}(w)$, and Faltings' topos associated to the pair of a log formal scheme and log rigid space: $(\mathcal{X}(w), X(w))$. Namely we first let $\mathcal{X}(w)^{\text {ket }}$ be the Kummer étale site of $\mathcal{X}(w)$, which is the full sub-category of the category of $\log$ schemes $\mathcal{T}$, endowed with a Kummer $\log$ étale morphism $\mathcal{T} \longrightarrow \mathcal{X}(w)$ (see $[\mathrm{AI}]$ section 2.2.1 or [Il] section 2.1). We recall that the fiber product in this category is the fiber product of log formal schemes in the category of fine and saturated log formal schemes so in particular the underlying formal scheme of the fiber product is not necessarily the fiber product of the underlying formal schemes (see [Ka]).

If $\mathcal{U}$ is an object in $\mathcal{X}(w)^{\text {ket }}$ then we denote by $\mathcal{U}_{\bar{K}}^{\text {fket }}$ the finite Kummer étale site attached to $\mathcal{U}$ over $\bar{K}$ as defined in $[\mathrm{AI}] \S 2.2 .2$.

Now, to define $\mathfrak{X}(w)$ we denote by $E_{\mathcal{X}(w)_{\bar{K}}}$ the category such that

i) the objects are pairs $(\mathcal{U}, W)$ such that $\mathcal{U} \in \mathcal{X}(w)^{\text {ket }}$ and $W \in \mathcal{U}_{\bar{K}}^{\text {fket }}$

ii) a morphism $\left(\mathcal{U}^{\prime}, W^{\prime}\right) \longrightarrow(\mathcal{U}, W)$ in $E_{\mathcal{X}(w)_{\bar{K}}}$ is a pair $(\alpha, \beta)$, where $\alpha: \mathcal{U}^{\prime} \longrightarrow \mathcal{U}$ is a morphism in $\mathcal{U}^{\text {ket }}$ and $\beta: W^{\prime} \longrightarrow W \times_{\mathcal{U}_{K}} \mathcal{U}_{K}^{\prime}$ is a morphism in $\left(\mathcal{U}^{\prime}\right)_{\bar{K}}^{\text {fket }}$.

The pair $(\mathcal{X}(w), X(w))$ is a final object in $E_{\mathcal{X}(w)_{\bar{K}}}$ and moreover in this category finite projective limits are representable and in particular fiber products exist (see [AI] section 2.2.3 and [Err] proposition 2.6 for an explicit description of the fiber product).

A family of morphisms $\left\{\left(\mathcal{U}_{i}, W_{i}\right) \longrightarrow(\mathcal{U}, W)\right\}_{i \in I}$ is a covering family if either

$(\alpha)\left\{\mathcal{U}_{i} \longrightarrow \mathcal{U}\right\}_{i \in I}$ is a covering family in $\mathcal{X}(w)^{\text {ket }}$ and $W_{i} \cong W \times \mathcal{U}_{K} \mathcal{U} i, K$ for every $i \in I$

or

$(\beta)$ there exists $\mathcal{U}$ in $\mathcal{X}(w)^{\text {ket }}$ such that $\mathcal{U}_{i} \cong \mathcal{U}$ for all $i \in I$ and $\left\{W_{i} \longrightarrow W\right\}_{i \in I}$ is a covering in $\mathcal{U}_{\bar{K}}^{\text {fket }}$.

We endow $E_{\mathcal{X}(w)_{\bar{K}}}$ with the topology generated by the covering families defined above and denote by $\mathfrak{X}(w)$ the associated site.

Let us observe that $\left(\mathcal{X}(w), X\left(p^{r}\right)(w)\right)$ is an object of $E_{\mathcal{X}(w)_{\bar{K}}}$, for all $r \geq 1$ (while $\left(\mathcal{X}\left(p^{r}\right)(w), X\left(p^{r}\right)(w)\right)$ is not!) so we define the induced (or localized) site $\mathfrak{X}\left(p^{r}\right)(w):=\mathfrak{X}(w) /\left(\mathcal{X}(w), X\left(p^{r}\right)(w)\right)$ see [AIS2] $\S 2.3,2.4,2.5$. 


\subsection{Sheaves on $\mathfrak{X}(w)$}

We denote by $\mathcal{O}_{\mathfrak{X}(w)}$ the presheaf of $\mathcal{O}_{\bar{K}}$-algebras on $\mathfrak{X}(w)$ defined by

$$
\mathcal{O}_{\mathfrak{X}}(\mathcal{U}, W):=\text { the normalization of } \mathrm{H}^{0}\left(\underline{\mathcal{U}}, \mathcal{O}_{\underline{\mathcal{U}}}\right) \text { in } \mathrm{H}^{0}\left(\underline{W}, \mathcal{O}_{\underline{W}}\right) \text {. }
$$

We also define by $\mathcal{O}_{\mathfrak{X}(w)}^{\text {un }}$ the sub-presheaf of $\mathbb{W}(k)$-algebras of $\mathcal{O}_{\mathfrak{X}(w)}$ whose sections over $(\mathcal{U}, W)$ consist of the elements $x \in \mathcal{O}_{\mathfrak{X}(w)}(\mathcal{U}, W)$ such that there exist a finite unramified extension $M$ of $K$ contained in $\bar{K}$, a Kummer log étale morphism $\mathcal{V} \longrightarrow \mathcal{U} \otimes_{\mathcal{O}_{K}} \mathcal{O}_{M}$ and a morphism $W \longrightarrow \mathcal{V}_{K}$ over $\mathcal{U}_{K}$ such that $x$, viewed as an element of $\mathrm{H}^{0}\left(\underline{W}, \mathcal{O}_{\underline{W}}\right)$ lies in the image of $\mathrm{H}^{0}\left(\underline{\mathcal{V}}, \mathcal{O}_{\underline{\mathcal{V}}}\right)$. Then the presheaves $\mathcal{O}_{\mathfrak{X}}$ and $\mathcal{O}_{\mathfrak{X}}^{\text {un }}$ are sheaves and $\mathcal{O}_{\mathfrak{X}}^{\text {un }}$ is isomorphic to the sheaf $v_{\mathcal{X}}^{*}\left(\mathcal{O}_{\mathcal{X}^{\text {ket }}}\right)$.

We denote by $\widehat{\mathcal{O}}_{\mathfrak{X}(w)}$ and $\widehat{\mathcal{O}}_{\mathfrak{X}}^{\text {un }}$ the continuous sheaves on $\mathfrak{X}$ given by the projective systems of sheaves $\left\{\mathcal{O}_{\mathfrak{X}} / p^{n} \mathcal{O}_{\mathfrak{X}}\right\}_{n \geq 0}$ and respectively $\left\{\mathcal{O}_{\mathfrak{X}}^{\text {un }} / p^{n} \mathcal{O}_{\mathfrak{X}}^{\text {un }}\right\}_{n \geq 0}$.

Let $r \geq 1$ and $w \in \mathbb{Q}$ adapted to $r$, let $Z:=X\left(p^{r}\right)(w) \longrightarrow X(w)$ and let us denote by $\mathfrak{X}\left(p^{r}\right)(w):=\mathfrak{X}_{/(\mathcal{X}(w), Z)}$ the site induced by $(\mathcal{X}, Z)$ and by $j_{r}^{*}, j_{r, *}\left(\cong j_{r, !}\right)$ the associated morphism of topoi. At the level of sites $j_{r}: \mathfrak{X}(w) \longrightarrow \mathfrak{X}\left(p^{r}\right)$ is defined by $j_{r}(\mathcal{U}, W):=\left(\mathcal{U}, Z \times_{X(w)} W, \operatorname{pr}_{1}\right)$ (see [AIS2] section 2.5).

We have functors

$$
v_{\mathfrak{X}(w)}: \mathcal{X}(w)^{\mathrm{ket}} \longrightarrow \mathfrak{X}(w), \quad v_{r}: \mathcal{X}(w)^{\text {ket }} \longrightarrow \mathfrak{X}\left(p^{r}\right)(w)
$$

defined by $v_{\mathfrak{X}(w)}(\mathcal{U}):=\left(\mathcal{U}, \mathcal{U}_{K}\right)$ and $v_{r}:=j_{r} \circ v_{\mathfrak{X}(w)}$. More explicitly, $v_{r}(\mathcal{U}):=j_{r}\left(\mathcal{U}, \mathcal{U}_{K}\right)=$ $\left(\mathcal{U}, Z \times_{X} \mathcal{U}_{K}, \mathrm{pr}_{1}\right)$. These functors send covering families to covering families, commute with fiber products and send final objects to final objects. In particular they define morphisms of topoi. The results of $\S 2$ of [AIS2] imply that the Leray spectral sequence for $v_{r, *}=v_{\mathfrak{X}(w), *} \circ j_{r, *}$ degenerates and we have $R^{i} v_{r, *} \cong R^{i} v_{\mathfrak{X}(w), *} \circ j_{r, *}$.

We denote by $\mathcal{O}_{\mathfrak{X}(r)(w)}:=j_{r}^{*}\left(\mathcal{O}_{\mathfrak{X}(w)}\right)$ and by $\widehat{\mathcal{O}}_{\mathfrak{X}(w)\left(p^{r}\right)}:=j_{r}^{*}\left(\widehat{\mathcal{O}}_{\mathfrak{X}(w)}\right)$. Let us recall the morphism $\theta_{r}: \mathcal{X}\left(p^{r}\right)(w) \longrightarrow \mathcal{X}(w)$ which is finite and defines $\mathcal{X}\left(p^{r}\right)(w)$ as the normalization of $\mathcal{X}(w)$ in $X\left(p^{r}\right)(w)$ and let $G_{r} \cong\left(\mathbb{Z} / p^{r} \mathbb{Z}\right)^{\times}$denote the Galois group of $X\left(p^{r}\right)(w) / X(w)$. Then $G_{r}$ acts naturally on $\mathcal{X}\left(p^{r}\right)(w)$ over $\mathcal{X}(w)$ and $\mathcal{X}(w) \cong \mathcal{X}\left(p^{r}\right)(w) / G_{r}$.

It is proved in [AIS2] that we have a natural isomorphism of sheaves on $\mathcal{X}(w)^{\text {ket }}$

$$
\left(v_{r, *}\left(\mathcal{O}_{\mathfrak{X}\left(p^{r}\right)(w)}\right)\right)^{G_{r}} \cong \mathcal{O}_{\mathcal{X}(w)} \text { and similarly }\left(v_{r, *}\left(\widehat{\mathcal{O}}_{\mathfrak{X}\left(p^{r}\right)(w)}\right)\right)^{G_{r}} \cong \widehat{\mathcal{O}}_{\mathcal{X}(w)} .
$$

\subsection{The localization functors}

We recall here the localization of a sheaf or a continuous sheaf on $\mathfrak{X}(w)$ to a "small affine of $\mathcal{X}^{\text {ket" }}$ (for more details see $[\mathrm{AI}]$ section 2.2.6). Let $\mathcal{U}=\left(\operatorname{Spf}\left(R_{\mathcal{U}}\right), N_{\mathcal{U}}\right)$ be a connected small affine object of $\mathcal{X}(w)^{\text {ket }}$ and we denote by $U:=\mathcal{U}_{K}$ the log rigid analytic generic fiber of $\mathcal{U}$. Let us recall that under the above hypothesis $\mathcal{U}$ is a log formal scheme whose log structure is given by the sheaf of monoids denoted $N_{\mathcal{U}}$.

We write $R_{\mathcal{U}} \otimes \bar{K}=\prod_{i=1}^{n} R_{\mathcal{U}, i}$ with $\operatorname{Spf}\left(R_{\mathcal{U}, i}\right)$ connected, we let $N_{\mathcal{U}, i}$ denote the monoids which give the respective $\log$ structures and we let $U_{i}$ denote the respective log rigid analytic generic fiber. Then each $R_{\mathcal{U}, i}$ is an integral domain, so we let $\mathbb{C}_{\mathcal{U}, i}$ denote an algebraic closure

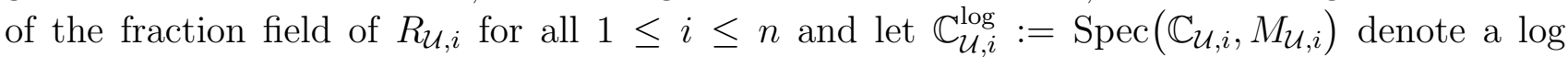
geometric point of $\left.\mathcal{U}_{i}:=\left(\operatorname{Spf}\left(R_{\mathcal{U}, i}\right), N_{\mathcal{U}, i}\right)\right)$ over $\mathbb{C}_{\mathcal{U}, i}$ (see [Il] definition 4.1 or [AI] section 2.2.5 for the definition of a $\log$ geometric point). We denote by $\mathcal{G}_{U, i}:=\pi_{1}^{\log }\left(U_{i}, \mathbb{C}_{\mathcal{U}, i}^{\log }\right)$ the Kummer 
étale fundamental group of $U_{i}$. We have then that the category $U_{i}^{\text {fket }}$ is equivalent to the category of finite sets with continuous $\mathcal{G}_{U, i}$-action. We write $\left(\bar{R}_{\mathcal{U}, i}, \bar{N}_{\mathcal{U}, i}\right)$ for the direct limit over all finite normal extensions $R_{\mathcal{U}, i} \subset S \subset \mathbb{C}_{\mathcal{U}, i}$, all log structures $N_{S}$ on $\operatorname{Spm}\left(S_{K}\right)$ such that there are Kummer étale morphisms $\mathbb{C}_{\mathcal{U}, i}^{\log } \longrightarrow\left(\operatorname{Spm}\left(S_{K}\right), N_{S}\right) \longrightarrow U_{i}$ compatible with the one between the underlying formal schemes. Finally we denote $\bar{R}_{\mathcal{U}}:=\prod_{i=1}^{n} \bar{R}_{\mathcal{U}, i}, \bar{N}_{\mathcal{U}}:=\prod_{i=1}^{n} \bar{N}_{\mathcal{U}, i}$ and $\mathcal{G}_{U_{\bar{K}}}:=\prod_{i=1}^{n} \mathcal{G}_{U, i}$.

We denote by $\operatorname{Rep}\left(\mathcal{G}_{U_{\bar{K}}}\right)$ and $\operatorname{Rep}\left(\mathcal{G}_{U_{\bar{K}}}\right)^{\mathbb{N}}$ the category of discrete abelian groups with continuous action by $\mathcal{G}_{U_{\bar{K}}}$, respectively the category of projective systems of such. It follows from [Il] section 4.5 that we have an equivalence of categories

$$
\operatorname{Sh}\left(U_{\bar{K}}^{\text {fket }}\right) \cong \operatorname{Rep}\left(\mathcal{G}_{U_{\bar{K}}}\right)
$$

sending $\mathcal{F} \rightarrow \lim _{\rightarrow} \mathcal{F}\left(\operatorname{Spm}\left(S_{K}\right), N_{S}\right)$. Therefore composing with the restriction $\operatorname{Sh}(\mathfrak{X}) \longrightarrow \operatorname{Sh}\left(U_{\bar{K}}^{\text {fket }}\right)$ defined by $\mathcal{F} \rightarrow(W \rightarrow \mathcal{F}(\mathcal{U}, W))$, we obtain a functor, called localization functor

$$
\operatorname{Sh}(\mathfrak{X}(w)) \longrightarrow \operatorname{Rep}\left(\mathcal{G}_{U_{\bar{K}}}\right) \text { denoted } \mathcal{F} \rightarrow \mathcal{F}\left(\bar{R}_{\mathcal{U}}, \bar{N}_{\mathcal{U}}\right)
$$

The importance of this result is given by the following result, see [AI] section 2.2.7:

Lemma 2.1. Let $\mathcal{F}$ be a locally free $\left(\widehat{\mathcal{O}}_{\mathfrak{X}(w)}\right)[1 / p]$-module of finite rank. The sheaf $R^{b} v_{\mathfrak{X}(w), *}(\mathcal{F})$ is the sheaf associated to the presheaf on $\mathcal{X}(w)^{\mathrm{ket}}$ :

$$
\mathcal{U}=\left(\operatorname{Spf}\left(R_{\mathcal{U}}\right), N_{\mathcal{U}}\right) \rightarrow \mathrm{H}^{b}\left(\mathcal{G}_{\mathcal{U}}, \mathcal{F}\left(\bar{R}_{\mathcal{U}}, \bar{N}_{\mathcal{U}}\right)\right)
$$

where $\mathcal{G}_{\mathcal{U}}$ is the Kummer-étale geometric fundamental group of $\mathcal{U}$, for a choice of a geometric generic point, i.e., $\mathcal{G}_{\mathcal{U}}=\operatorname{Gal}\left(\bar{R}_{\mathcal{U}}[1 / p] /\left(R_{\mathcal{U}} \bar{K}\right)\right)$.

We consider the following variant. Let $Z \rightarrow X(w)$ be a finite Kummer étale morphism in $X(w) \frac{\text { fket }}{K}$. Consider the associated site $\mathfrak{Z}:=\mathfrak{X}(w) /(\mathcal{X}(w), Z)$ and let $j_{(\mathcal{X}(w), Z)}: \mathfrak{X}(w) \rightarrow \mathfrak{Z}$ be the induced morphism of $\operatorname{sites} j_{(\mathcal{X}(w), Z)}(\mathcal{U}, W):=\left(\mathcal{U}, Z \times_{X(w)} W, \operatorname{pr}_{1}\right)$. Consider a sheaf $\mathcal{F} \in \operatorname{Sh}(\mathfrak{Z})$ and fix a connected small affine object $\mathcal{U}=\left(\operatorname{Spf}\left(R_{\mathcal{U}}\right), N_{\mathcal{U}}\right)$ of $\mathcal{X}(w)^{\text {ket }}$ as before. Denote by $\Upsilon_{\mathcal{U}}$ the set of homomorphisms of $R_{\mathcal{U}} \otimes \bar{K}$-algebras $\Gamma\left(Z \times_{X(w)} U, \mathcal{O}_{Z \times_{X(w)} U}\right) \rightarrow \bar{R}_{\mathcal{U}}[1 / p]$. For any $g \in \Upsilon_{\mathcal{U}}$ we write $\mathcal{F}\left(\bar{R}_{\mathcal{U}}, \bar{N}_{\mathcal{U}}, g\right):=\lim \mathcal{F}(\mathcal{U}, W)$, where the limit is taken over all finite and Kummer étale maps $\operatorname{Spm}\left(S_{K}\right)=W \rightarrow Z \times_{X(w)} U$ with $S_{K} \subset \bar{R}_{\mathcal{U}}[1 / p]$ a $\Gamma\left(Z \times_{X(w)} U, \mathcal{O}_{Z \times_{X} U}\right)$ subalgebra (using $g$ ). Let $\mathcal{G}_{U_{\bar{K}}, Z, g}$ be the subgroup of $\mathcal{G}_{U_{\bar{K}}}$ fixing $\Gamma\left(Z \times_{X(w)} U, \mathcal{O}_{Z \times_{X(w)} U}\right)$. Then $\operatorname{Sh}\left(\left(Z \times_{X(w)} U\right)^{\mathrm{fket}}\right) \cong \operatorname{Rep}\left(\mathcal{G}_{\mathcal{U}_{\bar{K}}, Z, g}\right)$ and we obtain as before a localization functor:

$$
\operatorname{Sh}(\mathfrak{Z}) \longrightarrow \operatorname{Rep}\left(\mathcal{G}_{\mathcal{U}_{\bar{K}}, Z, g}\right), \quad \mathcal{F} \rightarrow \mathcal{F}\left(\bar{R}_{\mathcal{U}}, \bar{N}_{\mathcal{U}}, g\right)
$$

If $\left\{\mathcal{U}_{i}\right\}_{i}$ is a covering of $\mathcal{X}^{\text {ket }}$ and for every $i$ we choose $g_{i} \in \Upsilon_{\mathcal{U}_{i}}$, it follows from the definition of coverings in the site $\mathfrak{Z}$ that the map $\operatorname{Sh}(\mathfrak{Z}) \longrightarrow \prod_{i} \operatorname{Rep}\left(\mathcal{G}_{U_{i, \bar{K}}, Z, g_{i}}\right)$ is faithful. It also follows from [AIS2] proposition 2.4 that

$$
j_{(\mathcal{X}, Z), *}(\mathcal{F})\left(\bar{R}_{\mathcal{U}}, \bar{N}_{\mathcal{U}}\right) \cong \oplus_{g \in \Upsilon_{\mathcal{U}}} \mathcal{F}\left(\bar{R}_{\mathcal{U}}, \bar{N}_{\mathcal{U}}, g\right)
$$

\section{Locally Analytic Modular Symbols}

\subsection{Accessible weights}

Let $\mathcal{W}$ denote the weight space for $\mathbf{G L}_{2 / \mathbb{Q}}$, i.e., the rigid analytic space over $\mathbb{Q}_{p}$ associated to the noetherian $\mathbb{Z}_{p}$-algebra $\mathbb{Z}_{p} \llbracket \mathbb{Z}_{p}^{\times} \rrbracket$. Let $k \in \mathcal{W}(K)$ be a $K$-valued weight, i.e., a continuous group 
homomorphism $k: \mathbb{Z}_{p}^{\times} \longrightarrow K^{\times}$. We embed $\mathbb{Z}$ in $\mathcal{W}\left(\mathbb{Q}_{p}\right)$ by sending $k \in \mathbb{Z}$ to the character $a \rightarrow a^{k}$ and in general if $k \in \mathcal{W}(K)$ as above and $t \in \mathbb{Z}_{p}^{\times}$we use the additive notation $t^{k}:=k(t)$.

Once we fixed $k \in \mathcal{W}(K)$ we define

$$
r=r(k):=\min \left\{n \in \mathbb{N} \quad \mid \quad n>0 \text { and }\left\|k\left(1+p^{n} \mathbb{Z}_{p}\right)-1\right\|<p^{\frac{-1}{p-1}}\right\}
$$

and we choose $w \in \mathbb{Q}$ with $w \geq 0$ and $w<2 /\left(p^{r}-1\right)$ if $p>3$ and $w<1 / 3^{r}$ if $p=3$. We say that $w$ is adapted to $r$ (and to $k$ ). Let us also note that given $k, r$ as above there is a unique $a \in K$ such that for all $t \in 1+p^{r} \mathbb{Z}_{p}$ we have $t^{k}=\exp (a \log (t))$.

Denote by $\mathcal{W}^{*}$ the rigid subspace of $\mathcal{W}$ of weights $k$ such that $\left|k(t)^{p-1}-1\right|<p^{-1 /(p-1}$, i.e., of weights $k$ such that $r(k)=1$. we call it the subspace of accessible weights.

\subsection{Analytic functions and distributions}

In this section we recall a number of definitions and results from [AIS2] and also from [AS] and [HIS]. Let $T_{0}:=\mathbb{Z}_{p}^{\times} \times \mathbb{Z}_{p}$, which we regard as a compact open subset of the space of row vectors $\left(\mathbb{Z}_{p}\right)^{2}$. We have the following structures on $T_{0}$ :

a) a natural left action of $\mathbb{Z}_{p}^{\times}$by scalar multiplication;

b) a natural right action of the semigroup

$$
\Xi\left(\mathbb{Z}_{p}\right)=\left\{\left(\begin{array}{ll}
a & b \\
c & d
\end{array}\right) \in M_{2}\left(\mathbb{Z}_{p}\right) \mid(a, c) \in \mathbb{Z}_{p}^{\times} \times p \mathbb{Z}_{p}\right\}
$$

and its subgroup

$$
\operatorname{Iw}\left(\mathbb{Z}_{p}\right):=\Xi\left(\mathbb{Z}_{p}\right) \cap \mathbf{G L}\left(\mathbb{Z}_{p}\right)
$$

given by matrix multiplication on the right.

The two actions commute.

Definition 3.1. We set

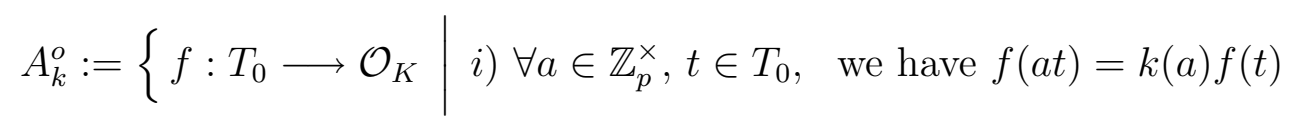

and

ii) the function $z \rightarrow f(1, z)$ extends to a rigid analytic function on the closed unit disk $B[0,1]\}$.

Let us make ii) of the above definition more precise. Let $\operatorname{ord}_{p}$ denote the $p$-adic valuation on $\mathcal{O}_{K}$ normalized such that $\operatorname{ord}_{p}(p)=1$. Then we say that the function $z \rightarrow f(1, z)$ in the definition above is rigid analytic on $B[0,1]$ if there exists a power series $F(X)=\sum_{n=0}^{\infty} a_{n} X^{n} \in \mathcal{O}_{K} \llbracket X \rrbracket$ with $\operatorname{ord}_{p}\left(a_{n}\right) \stackrel{n \rightarrow \infty}{\longrightarrow} \infty$ and such that $f(1, z)=F(z)$ for all $z \in \mathbb{Z}_{p}$. Let us denote by $A_{k}:=A_{k}^{o} \otimes_{\mathcal{O}_{K}} K$, which is naturally a $K$-vector space. In fact $A_{k}$ is an orthonormalizable $K$-Banach space, where an orthonormal basis is given by: $\left\{f_{n}\right\}_{n \geq 0}$ where $f_{n} \in A_{k}^{o}$ are the unique elements such that $f_{n}(1, z)=z^{n}$ for all $z \in \mathbb{Z}_{p}$. In other words $f_{n}(x, y)=k(x)(y / x)^{n}$ for all $(x, y) \in T_{0}$.

For every $\gamma \in \Xi\left(\mathbb{Z}_{p}\right)$ and function $f: T_{0} \longrightarrow \mathcal{O}_{K}$ we define $(\gamma f)(v):=f(v \gamma)$. We have

Lemma 3.2. If $f \in A_{k}^{o}$ and $\gamma \in \Xi\left(\mathbb{Z}_{p}\right)$ then $\gamma f \in A_{k}^{o}$. 
Proof. Let $\gamma=\left(\begin{array}{ll}a & b \\ c & d\end{array}\right)$. Then for every $v \in T_{0}$ and $a \in \mathbb{Z}_{p}^{\times}$we have $(\gamma f)(a v)=f((a v) \gamma)=$ $k(a) f(v \gamma) k(a)(\gamma f)(v)$.

Moreover,

$$
(\gamma f)(1, z)=f(a+c z, b+d z)=k(a+c z) f\left(1, \frac{b+d z}{a+c z}\right)=k(a) k\left(1+c a^{-1} x\right) \sum_{n=0}^{\infty} a_{n}\left(\frac{b+d z}{a+c z}\right)^{n} .
$$

Using the fact that $k$ is analytic and $a_{n} \stackrel{n \rightarrow \infty}{\longrightarrow} 0$ we deduce that the function $z \rightarrow(\gamma f)(1, z)$ extends to an analytic function on the closed unit disk $B[0,1]$.

Definition 3.3. Let $k \in \mathcal{W}^{*}(K)$ be a weight. We define $D_{k}^{o}:=\operatorname{Hom}_{\text {cont }, \mathcal{O}_{\mathrm{K}}}\left(A_{k}^{o}, \mathcal{O}_{K}\right)$, i.e., the $\mathcal{O}_{K}$-module of continuous, $\mathcal{O}_{K}$-linear homomorphisms from $A_{k}^{o}$ to $\mathcal{O}_{K}$. We also denote by $D_{k}:=D_{k}^{o} \otimes_{\mathcal{O}_{K}} K$.

Remark 3.4. i) The (left) action of the semigroup $\Xi\left(\mathbb{Z}_{p}\right)$ on $A_{k}^{o}$ induces a (right) action on $D_{k}^{o}$ by $(\mu \mid \gamma)(f):=\mu(\gamma f)$ for all $\gamma \in \Xi\left(\mathbb{Z}_{p}\right), f \in A_{k}^{o}$ and $\mu \in D_{k}^{o}$.

ii) It is customary to define $D_{k}$ by taking the continuous and compact homomorphisms from $A_{k}^{o}$ to $\mathcal{O}_{K}$. Our definition has the advantage that the cohomology of $D_{k}$ with respect to modular groups can be naturally identified with the étale cohomology of lisse sheaves on modular curves and, in particular, inherits an action of the absolute Galois group of $\mathbb{Q}$. We refer to the introduction of [AIS2] for a discussion.

iii) We have a natural, fundamental homomorphism of $\mathcal{O}_{K}$-modules

$$
\psi: D_{k}^{o} \longrightarrow \prod_{n \in \mathbb{N}} \mathcal{O}_{K}, \text { defined by } \mu \rightarrow\left(\mu\left(f_{n}\right)\right)_{n \in \mathbb{N}} .
$$

As the family $\left(f_{n}\right)_{n}$ is an orthonormal basis of $A_{k}$ over $K$, the above morphism is a $\mathcal{O}_{K}$-linear isomorphism. Moreover, under this isomorphism, the weak topology on $D_{k}^{o}$ corresponds to the weak topology (i.e., the product of the $\underline{m}_{K}$-adic topologies on the product where $\underline{m}_{K}$ is the maximal ideal of $\left.\mathcal{O}_{K}\right)$.

\subsection{The geometric picture}

Let us recall from the beginning of section $\S 2.1$ the modular curves $X(N, p) \longrightarrow X_{1}(N)$, their natural formal models $\mathcal{X}(N, p) \longrightarrow \mathcal{X}_{1}(N)$ and if $w \in \mathbb{Q}, 0 \leq w \leq p /(p+1)$ we also have a rigid analytic space $X(w) \subset X(N, p)$ and its formal model $\mathcal{X}(w)$, with its natural morphism $\mathcal{X}(w) \longrightarrow \mathcal{X}(N, p)$. All these rigid spaces and formal schemes are in fact log rigid spaces and respectively log formal schemes, which are all log smooth and all the maps described are maps of $\log$ formal schemes or log rigid spaces.

Sheaves on $X(N, p) \frac{\text { ket }}{K}$ associated to analytic distributions: Let $\mathcal{E} \longrightarrow X(N, p)$ be the universal generalised elliptic curve, and let us denote by $\mathcal{T}$ the $p$-adic Tate-module of $\mathcal{E}$, seen as a continuous sheaf on the Kummer étale site of $X(N, p)$, denoted $X(N, p)^{\text {ket }}$. If $\eta=\operatorname{Spec}(\mathbb{K})$ denotes a geometric generic point of $X(N, p)$, let $\mathcal{G}$ denote the geometric Kummer étale fundamental group associated to $(X(N, p), \eta)$ and let $T:=\mathcal{T}_{\eta}$. One can easily see that $T$ is a free $\mathbb{Z}_{p}$-module of rank 2 with continuous action of $\mathcal{G}$. Let us choose a $\mathbb{Z}_{p}$-basis $\left\{\epsilon_{0}, \epsilon_{1}\right\}$ of $T$ satisfying the properties: $\left\langle\epsilon_{0}, \epsilon_{1}\right\rangle=1, \epsilon_{0}(\bmod p T) \in \mathcal{E}_{\eta}[p]$ does not belong to the universal level $p$-subgroup $C$ and $\epsilon_{1}(\bmod p T)$ is a generator of $C$. We let $T_{0}:=\left\{a \epsilon_{0}+b \epsilon_{1} \quad \mid a \in \mathbb{Z}_{p}^{*}, b \in \mathbb{Z}_{p}\right\}$. Then $T_{0}$ 
is a compact subset of $T$ preserved by $\mathcal{G}$ which can be identified to $\mathbb{Z}_{p}^{\times} \times \mathbb{Z}_{p}$. Moreover the right action of $\mathcal{G}$ on the above chosen basis defines a continuous group homomorphism

$$
\gamma: \mathcal{G} \longrightarrow \text { Iw defined by }\left(\epsilon_{0} \sigma, \epsilon_{1} \sigma\right)=\left(\epsilon_{0}, \epsilon_{1}\right) \gamma(\sigma) \text { for } \sigma \in \mathcal{G} \text {. }
$$

Therefore, if $k \in \mathcal{W}^{*}$ and $n \geq 1$ as in section 3.2 then via the homomorphism $\gamma$ above, the Iw-modules $A_{k}$ and $D_{k}$ can be seen as ind-continuous representations of $\mathcal{G}$.

Notation: For later use, for $A=A_{k}$ we write $\mathcal{A}^{o}:=\left(\mathcal{A}_{n}^{o}\right)_{n \in \mathbb{N}}$ for the continuous sheaf on Faltings' site $\mathfrak{X}(N, p)$ associated to the continuous representation of $A^{o}=\left(A^{o} / \underline{m}_{K}^{n} A^{o}\right)_{n \in \mathbb{N}}$ of the Kummer étale fundamental group $\mathcal{G}$ of $X(N, p)$. The ind-continuous sheaf $\mathcal{A}$ is simply $\mathcal{A}^{o}[1 / p]$.

Analogously, for $D_{k}$, we write $\mathcal{D}_{k}^{o}:=\left(\mathcal{D}_{k, n}\right)_{n \in \mathbb{N}}$ for the continuous sheaf associated to $D_{k}^{o} / \operatorname{Fil}^{n}\left(D_{k}^{o}\right)$. Then, $\mathcal{D}_{k}$ is the ind-continuous sheaf $\mathcal{D}_{k}^{o}[1 / p]$.

Sheaves on Faltings' site associated to analytic distributions: Let us now denote by $\mathfrak{X}(w)$ Faltings' site associated to the pair $(\mathcal{X}(w), X(w))$. The map of sites $u: \mathfrak{X}(w) \longrightarrow X(w) \frac{\text { ket }}{K}$, given by $(U, W) \mapsto W$, sends covering families to covering families, commutes with fibre products and sends the final object to the final object. It defines a morphism of topoi $u_{*}: \operatorname{Sh}\left(X(w) \frac{\text { ket }}{K}\right) \longrightarrow$ $\operatorname{Sh}(\mathfrak{X}(w))$ which extends to inductive systems of continuous sheaves. In particular all the indcontinuous Kummer étale sheaves $\mathcal{D}_{k}$ can be seen as ind-continuous Kummer étale sheaves on $X(N, p) \frac{\text { ket }}{K}$, by restriction as ind-continuous Kummer étale sheaves on $X(w) \frac{\text { ket }}{K}$ and by applying $u_{*}$ as ind-continuous sheaves on $\mathfrak{X}(w)$. For simplicity we omit $u_{*}$ from the notations.

\section{Modular sheaves}

\subsection{The sheaves $\Omega_{\mathfrak{X}}^{k}(w)$}

In this section we recall the main constructions of chapter 3 of [AIS2]. Let $w \in \mathbb{Q}$ be such that $0 \leq w<p /(p+1)$.

Let $f: \mathcal{E} \longrightarrow \mathcal{X}(w)$ and $f_{K}: \mathcal{E}_{K} \longrightarrow X(w)$ denote the universal semi-abelian scheme over $\mathcal{X}(w)$ and respectively its generic fiber and let $\mathcal{T} \longrightarrow X(w)$ denote the $p$-adic Tate module of $\mathcal{E}_{K}^{\vee} \longrightarrow X(w)$ seen as a continuous sheaf on $\mathcal{X}(w) \frac{\text { ket }}{K}$. Notice that $\mathcal{E}$ admits a canonical subgroup $\mathcal{C}_{1} \subset \mathcal{E}$. Let $\mathcal{T}_{0} \subset \mathcal{T}$ be the inverse image of $\mathcal{C}_{1, K}^{\vee} \backslash\{0\}$ in $\mathcal{T}$ via the natural map $\mathcal{T} \rightarrow \mathcal{E}_{K}^{\vee}[p] \rightarrow \mathcal{C}_{1, K}^{\vee}$. Then $\mathcal{T}$ and $\mathcal{T}_{0}$ are continuous, locally constant sheaves on $\mathcal{X}(w) \frac{\text { ket }}{K}$ and as such can be seen as a continuous sheaf on $\mathfrak{X}(w)$. Notice that the sheaf $\mathcal{T}$ is a continuous sheaf of abelian groups, while $\mathcal{T}_{0}$ is a continuous sheaves of sets and it is endowed with a natural action of $\mathbb{Z}_{p}^{*}$.

Let $e: \mathcal{X}(w) \longrightarrow \mathcal{E}$ denote the identity section of $f$ and let $\omega_{\mathcal{E} / \mathcal{X}(w)}:=e^{*}\left(\Omega_{\mathcal{E} / \mathcal{X}(w)}^{1}\right)$. It is a locally free $\mathcal{O}_{\mathcal{X}(w)}$-module of rank 1 and we denote by $\omega_{\mathcal{E} / \mathfrak{X}(w)}:=v_{\mathcal{X}(w)}^{*}\left(\omega_{\mathcal{E} / \mathcal{X}(w)}\right)$. Then $\omega_{\mathcal{E} / \mathfrak{X}(w)}$ is a continuous sheaf on $\mathfrak{X}(w)$, a locally free $\widehat{\mathcal{O}}_{\mathfrak{X}(w)}^{\text {un }}$-module of rank 1 .

We have a natural sequence of sheaves and morphisms of sheaves on $\mathfrak{X}(w)$ called the HodgeTate sequence of sheaves for $\mathcal{E} / \mathcal{X}(w)$

$$
0 \longrightarrow \omega_{\mathcal{E} / \mathfrak{X}(w)}^{-1} \otimes_{\widehat{\mathcal{O}}_{\mathfrak{X}(w)}^{\text {un }}} \widehat{\mathcal{O}}_{\mathfrak{X}(w)}(1) \longrightarrow \mathcal{T} \otimes \widehat{\mathcal{O}}_{\mathfrak{X}(w)} \stackrel{\operatorname{dlog}}{\longrightarrow} \omega_{\mathcal{E} / \mathfrak{X}(w)} \otimes_{\widehat{\mathcal{O}}_{\mathfrak{X}(w)}^{u n}} \widehat{\mathcal{O}}_{\mathfrak{X}(w)} \longrightarrow 0
$$


For every connected, small affine object $\mathcal{U}=\left(\operatorname{Spf}\left(R_{\mathcal{U}}\right), N_{\mathcal{U}}\right)$ of $\mathcal{X}(w)^{\text {ket }}$, the localization of the Hodge-Tate sequence (2) at $\mathcal{U}$ gives the Hodge-Tate sequence of continuous $\mathcal{G}_{\mathcal{U}}$-representations which appears in [AIS1] section 2

$$
0 \longrightarrow \omega_{\mathcal{E} / \mathcal{X}(w)}^{-1}(\mathcal{U}) \otimes_{R_{\mathcal{U}}} \widehat{\bar{R}}_{\mathcal{U}}(1) \longrightarrow T_{p}\left(\mathcal{E}_{\mathcal{U}}^{\vee}\right) \otimes \widehat{\bar{R}}_{\mathcal{U}} \stackrel{\operatorname{dlog} \mathcal{U}}{\longrightarrow} \omega_{\mathcal{E} / \mathcal{X}(w)}(\mathcal{U}) \otimes_{R_{\mathcal{U}}} \widehat{\bar{R}}_{\mathcal{U}} \longrightarrow 0
$$

Moreover, in [AIS2] it is proved the following. Let $\mathcal{F}^{0}:=\operatorname{Im}(\operatorname{dlog})$ and $\mathcal{F}^{1}:=\operatorname{Ker}(\operatorname{dlog})$. Then

i) $\mathcal{F}^{0}, \mathcal{F}^{1}$ are locally free sheaves of $\widehat{\mathcal{O}}_{\mathfrak{X}(w)}$-modules on $\mathfrak{X}(w)$ of rank 1 . We denote by $\mathcal{F}^{i,(r)}:=$ $j_{r}^{*}\left(\mathcal{F}^{i}\right)$ for $i=0,1$, they are locally free $\widehat{\mathcal{O}}_{\mathfrak{X}\left(p^{r}\right)(w)}$-modules of rank 1 .

ii) We set $v:=w /(p-1)$ and let us suppose that $w$ is adapted to $r$, for a certain $r \geq 1$, $r \in \mathbb{N}$. We denote $\mathcal{C}_{r} \subset \mathcal{E}\left[p^{r}\right]$ the canonical subgroup of level $p^{r}$ of $\mathcal{E}\left[p^{r}\right]$ over $X\left(p^{r}\right)(w)$ (which exists by the assumption on $w$ ), denote by $\mathcal{C}_{r}^{\vee}$ its Cartier dual and we also denote by $\mathcal{C}_{r}$ and $\mathcal{C}_{r}^{\vee}$ the groups of points of these group-schemes over $X\left(p^{r}\right)(w)$, and by the same symbols the constant abelian sheaves on $\left(X\left(p^{r}\right)(w)\right)^{\text {ket }}$. We have natural isomorphisms as $\widehat{\mathcal{O}}_{\mathfrak{X}(w)}$-modules on $\mathfrak{X}(w): \mathcal{F}^{0} / p^{(1-v) r} \mathcal{F}^{0} \cong \mathcal{C}_{r}^{\vee} \otimes \mathcal{O}_{\mathfrak{X}(w)} / p^{(1-v) r} \mathcal{O}_{\mathfrak{X}(w)}$ and $\mathcal{F}^{1} / p^{(1-v) r} \mathcal{F}^{1} \cong \mathcal{C}_{r} \otimes \mathcal{O}_{\mathfrak{X}(w)} / p^{(1-v) r} \mathcal{O}_{\mathfrak{X}(w)}$.

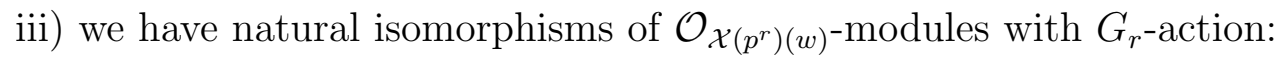

$$
v_{\mathfrak{X}\left(p^{r}\right)(w), *}\left(\mathcal{F}^{i,(r)}\right) \cong \mathcal{F}_{i}^{(r)} \otimes_{\mathcal{O}_{K}} \mathcal{O}_{\mathbb{C}_{p}}
$$

for $i=0,1$. Here $\mathcal{F}_{i}^{(r)}$ are the sheaves on $\mathcal{X}\left(p^{r}\right)(w)$ defined in section 2 of [AIS1]. Moreover $\mathcal{F}^{i,(r)} \cong v_{\mathfrak{X}\left(p^{r}\right)(w)}^{*}\left(\mathcal{F}_{i}^{(r)}\right) \otimes_{\widehat{\mathcal{O}}_{\mathfrak{X}(p)}^{\mathrm{un}}\left(p^{r}\right)(w)} \widehat{\mathcal{O}}_{\mathfrak{X}\left(p^{r}\right)(w)}$.

Let $k \in \mathcal{W}(K)$ and $r \in \mathbb{N}$ and $w \in \mathbb{Q}$ such that $w$ is adapted to $r$ and $k$. Let us also recall that we denoted $v:=w /(p-1)$.

Let us denote $S_{\mathfrak{X}\left(p^{r}\right)(w)}:=\mathbb{Z}_{p}^{\times}\left(1+p^{(1-v) r} \widehat{\mathcal{O}}_{\mathfrak{X}\left(p^{r}\right)(w)}\right)$, it is a sheaf of abelian groups on $\mathfrak{X}\left(p^{r}\right)(w)$ which acts on $\widehat{\mathcal{O}}_{\mathfrak{X}\left(p^{r}\right)(w)}$ as follows: let $s=c \cdot x \in S_{\mathfrak{X}\left(p^{r}\right)(w)}(\mathcal{U}, W, \alpha)=\mathbb{Z}_{p}^{\times}\left(1+p^{(1-v) r} \widehat{\mathcal{O}}_{\mathfrak{X}(w)}(\mathcal{U}, W)\right)$ and $y \in \widehat{\mathcal{O}}_{\mathfrak{X}\left(p^{r}\right)(w)}(\mathcal{U}, W, \alpha)=\widehat{\mathcal{O}}_{\mathfrak{X}(w)}(\mathcal{U}, W)$. Then we define

$$
s * y:=\exp (a \log (x)) \cdot c^{k} \cdot y, \text { where } a \in K \text { is such that } t^{k}=\exp (a \log (t)), t \in 1+p^{r} \mathbb{Z}_{p} .
$$

Let us remark that $s * y \in \widehat{\mathcal{O}}_{\mathfrak{X}\left(p^{r}\right)(w)}(\mathcal{U}, W, \alpha)$. We denote by $\left(\widehat{\mathcal{O}}_{\mathfrak{X}\left(p^{r}\right)(w)}\right)^{(k)}$ the continuous sheaf $\widehat{\mathcal{O}}_{\mathfrak{X}\left(p^{r}\right)(w)}$ with the above defined action of $S_{\mathfrak{X}\left(p^{r}\right)(w)}$.

We have an isomorphism of sheaves

$$
\varphi: \mathcal{F}^{0,(r)} / p^{(1-v) r} \mathcal{F}^{0,(r)} \cong\left(\mathcal{C}_{r}\right)^{\vee} \otimes \widehat{\mathcal{O}}_{\mathfrak{X}\left(p^{r}\right)(w)} / p^{(1-v) r} \widehat{\mathcal{O}}_{\mathfrak{X}\left(p^{r}\right)(w)} .
$$

Let $\mathcal{F}^{(r)^{\prime}}$ denote the inverse image under the isomorphism $\varphi$ above of the sheaf of sets $\left(\mathcal{C}_{r}\right)^{\vee}-$ $\left(\mathcal{C}_{r}\right)^{\vee}\left[p^{r-1}\right]$. It is endowed with an action of $S_{\mathfrak{X}\left(p^{r}\right)(w)}$.

Recall that we have a morphism of sites $j_{r}: \mathfrak{X}(w) \rightarrow \mathfrak{X}\left(p^{r}\right)(w)$. It then follows from the construction that dlog induces a map

$$
\operatorname{dlog}: j_{r}^{*}\left(\mathcal{T}_{0}\right) \longrightarrow \mathcal{F}^{(r)^{\prime}}
$$

compatible with the actions of $\mathbb{Z}_{p}^{*}$ on the two sides.

Moreover, following [AIS2] $\S 4$, the sheaf $\mathcal{F}^{(r)^{\prime}}$ is an $S_{\mathfrak{X}\left(p^{r}\right)(w)}$-torsor and there exists a covering of $\mathcal{X}(w)$ by small affine objects $\left\{U_{i}\right\}$ such that $\left.\mathcal{F}^{(r)^{\prime}}\right|_{\left(U_{i}, U_{i} \times \mathcal{X}(w)\right.} X\left(p^{r}\right)$ is the trivial torsor for every $i$. 
Let us now consider the $\mathcal{O}_{\mathfrak{X}\left(p^{r}\right)(w)}$-module

$$
\mathcal{M}_{k}^{(r)}(w):=\mathfrak{H}_{o} m_{S_{\mathfrak{X}\left(p^{r}\right)(w)}}\left(\mathcal{F}^{(r)^{\prime}},\left(\widehat{\mathcal{O}}_{\mathfrak{X}\left(p^{r}\right)(w)}\right)^{(-k)}\right) .
$$

It is a locally free $\mathcal{O}_{\mathfrak{X}\left(p^{r}\right)(w)}$-module of rank 1 and we have a natural isomorphism of $\mathcal{O}_{\mathfrak{X}\left(p^{r}\right)(w)^{-}}$ modules

$$
\mathfrak{H o m}_{\widehat{\mathcal{O}}_{\mathfrak{X}\left(p^{r}\right)(w)}}\left(\mathcal{M}_{k}^{(r)}(w), \widehat{\mathcal{O}}_{\mathfrak{X}\left(p^{r}\right)(w)}\right) \longrightarrow \mathcal{M}_{-k}^{(r)}(w) .
$$

We also have a map of continuous sheaves of $\mathcal{O}_{\mathfrak{X}\left(p^{r}\right)(w) \text {-modules }}$

$$
\operatorname{dlog}{ }^{\vee, k}: \mathcal{M}_{k}^{(r)}(w) \longrightarrow \mathfrak{H} o m_{\mathbb{Z}_{p}^{*}}\left(j_{r}^{*}\left(\mathcal{T}_{0}\right),\left(\widehat{\mathcal{O}}_{\mathfrak{X}\left(p^{r}\right)(w)}\right)^{(-k)}\right)
$$

induced by dlog.

For every element $\sigma \in G_{r}$ we denote also by $\sigma$ the functor $\left(E_{\mathcal{X}(w)_{K}}\right)_{/\left(\mathcal{X}(w), X\left(p^{r}\right)(w)\right)} \longrightarrow$ $\left(E_{\mathcal{X}(w)_{\bar{K}}}\right)_{/\left(\mathcal{X}(w), X\left(p^{r}\right)(w)\right)}$ defined on objects by $(\mathcal{U}, W, \alpha) \rightarrow(\mathcal{U}, W, \sigma \circ \alpha)$ and by identity on the morphisms. This functor induces a continuous functor on the site $\mathfrak{X}\left(p^{r}\right)(w)$. If $\mathcal{H}$ is a sheaf (or continuous sheaf) on $\mathfrak{X}\left(p^{r}\right)(w)$ we denote by $\mathcal{H}^{\sigma}$ the sheaf: $\mathcal{H}^{\sigma}(\mathcal{U}, W, \alpha):=\mathcal{H}(\sigma(\mathcal{U}, W, \alpha))=$ $\mathcal{H}(\mathcal{U}, W, \sigma \circ \alpha)$.

a) Let us suppose that $\mathcal{G}$ is a sheaf of abelian groups on $\mathfrak{X}(w)$ and $\mathcal{H}:=j_{r}^{*}(\mathcal{G})$. Then $\mathcal{H}^{\sigma}=\mathcal{H}$ for all $\sigma \in G_{r}$.

b) For $\mathcal{H}=\mathcal{F}^{(r)^{\prime}},\left(\widehat{\mathcal{O}}_{\mathfrak{X}\left(p^{r}\right)(w)} \hat{\otimes} B\right)^{(-k)}$, or $j_{r}^{*}\left(\mathcal{T}_{0}\right)$ we have $(\mathcal{H})^{\sigma}=\mathcal{H}$ for every $\sigma \in G_{r}$. Hence, the same applies for $\mathcal{M}_{k}^{(r)}(w)$ and $\mathbb{A}_{k}^{(r)}$ compatibly with dlog ${ }^{\vee, k}$.

c) Suppose that $\mathcal{H}$ is a sheaf on $\mathfrak{X}\left(p^{r}\right)(w)$ such that $\mathcal{H}^{\sigma}=\mathcal{H}$ for all $\sigma \in G_{r}$. Then each element $\sigma \in G_{r}$ defines a canonical automorphism of the sheaf $j_{r, *}(\mathcal{H})$, i.e., we have a canonical action of the group $G_{r}$ on the sheaf $j_{r, *}(\mathcal{H})$.

Definition 4.1. We define the sheaves $\Omega_{\mathfrak{X}(w)}^{k}$ and $\omega_{\mathfrak{X}(w)}^{\dagger, k}$ on $\mathfrak{X}(w)$ by

$$
\Omega_{\mathfrak{X}(w)}^{k}:=\left(j_{r, *}\left(\mathfrak{H} o m_{S_{\mathfrak{X}\left(p^{r}\right)(w)}}\left(\mathcal{F}^{(r)^{\prime}},\left(\widehat{\mathcal{O}}_{\mathfrak{X}\left(p^{r}\right)(w)}\right)^{(-k)}\right)\right)\right)^{G_{r}}
$$

and

$$
\omega_{\mathfrak{X}(w)}^{\dagger, k}:=\left(j_{r, *}\left(\mathfrak{H} o m_{S_{\mathfrak{X}\left(p^{r}\right)(w)}}\left(\mathcal{F}^{(r)^{\prime}},\left(\widehat{\mathcal{O}}_{\mathfrak{X}\left(p^{r}\right)(w)}\right)^{(-k)}\right)\right)[1 / p]\right)^{G_{r}} .
$$

The sheaves thus defined enjoy the following properties. For every $k$ and $w$ as above we have i) $\omega_{\mathfrak{X}(w)}^{\dagger, k}$ is a locally free $\left(\widehat{\mathcal{O}}_{\mathfrak{X}(w)}\right)[1 / p]$-module of rank 1 .

ii) $v_{\mathfrak{X}(w), *}\left(\omega_{\mathfrak{X}}^{\dagger, k}\right) \cong \omega_{w}^{\dagger, k} \otimes_{K} \mathbb{C}_{p}$, where $\omega_{w}^{\dagger, k}$ is the invertible $\widehat{\mathcal{O}}_{\mathcal{X}(w)}[1 / p]$-module given in section 3.3 of [AIS1].

iii) $\omega_{\mathfrak{X}(w)}^{\dagger, k} \cong \omega_{w}^{\dagger, k} \hat{\otimes}_{\widehat{\mathcal{O}}_{\mathcal{X}(w)}} \widehat{\mathcal{O}}_{\mathfrak{X}(w)}$.

Remark 4.2. We remark that for $r=1$ the properties above remain true without inverting $p$ and for $\Omega_{\mathfrak{X}(w)}^{\dagger, k}$ in place of $\omega_{\mathfrak{X}(w)}^{\dagger, k}$ and for an invertible $\widehat{\mathcal{O}}_{\mathcal{X}(w)}$-module $\Omega_{w}^{\dagger, k}$ in place of $\omega_{w}^{\dagger, k}$.

This follows as $G_{1}=(\mathbb{Z} / p \mathbb{Z})^{*}$ is a group of order prime to $p$ so that taking $G_{1}$-invariants is a well behaved operation. See [AIS1] corollary 3.17. This will play a crucial role in the next section. 


\subsection{The sheaves $\mathbb{D}_{k}$, and $\mathbb{D}_{k}^{m}$.}

We start by fixing $k, w$ as in the previous section with $k$ accessible. The goal of this section is to construct a decreasing and exhausting filtration of the sheaf $\mathcal{D}_{k}^{o} \hat{\otimes} \mathcal{O}_{\mathfrak{X}(w)}$ by $\widehat{\mathcal{O}}_{\mathfrak{X}(w)^{-}}$-sub modules, denoted $\left(\mathbb{D}_{k}^{m}\right)_{m \geq-1}$ and determine their graded quotients. In fact we will proceed as follows: we will first define (independently) the sheaves $\operatorname{fil}_{m}\left(\mathbb{A}_{k}\right), \mathbb{D}_{k}^{m}$, for $m \geq-1$ and we will show that $\mathbb{D}_{k}^{-1} \cong \mathcal{D}_{k}^{o} \hat{\otimes} \mathcal{O}_{\mathfrak{X}(w)}$ as $\widehat{\mathcal{O}}_{\mathfrak{X}(w)}$-modules.

Let $m, n \geq 0$ be integers. We define

$$
\operatorname{fil}_{m}\left(\mathbb{A}_{k}\right):=\operatorname{Sym}^{m}\left(\mathcal{T}^{\vee}\right) \otimes_{\mathbb{Z}_{p}} \Omega_{\mathfrak{X}(w)}^{-k+m}
$$

Then $\operatorname{fil}_{m}\left(\mathbb{A}_{k}\right)$ is a locally free $\widehat{\mathcal{O}}_{\mathfrak{X}(w)}$-module of rank $m+1$ thanks to remark 4.2 .

Let $\mathcal{U}:=\left(\operatorname{Spf}\left(R_{\mathcal{U}}\right), N_{\mathcal{U}}\right)$ denote a connected, small object of $\mathcal{X}(w)^{\text {ket }}$, let $T:=\mathcal{T}\left(\bar{R}_{\mathcal{U}}, \bar{N}_{\mathcal{U}}\right)$ be the $p$-adic Tate module of $\mathcal{E}_{R_{\mathcal{U}}} \longrightarrow \operatorname{Spec}\left(R_{\mathcal{U}}\right)$ and let $C \subset \mathcal{E}[p]$ denote the canonical subgroup. We let $e_{0}, e_{1}$ denote an $\widehat{R}_{\mathcal{U}}$-basis of $T \otimes \widehat{\bar{R}}_{\mathcal{U}}$ such that $e_{1}$ is a basis of $F^{1}$ over $\widehat{\bar{R}}_{\mathcal{U}}$ whose restriction to $X(p)(w)$ reduces to the canonical generator of $\mathcal{C}$ modulo $p^{1-v}, \operatorname{dlog}_{\mathcal{U}}\left(e_{0}\right)$ is a basis of $F^{0}$ over $\widehat{\bar{R}}_{\mathcal{U}}$ whose restriction to $X(p)(w)$ reduces to the dual generator of $\mathcal{C}^{\vee}$ modulo $p^{1-v}$. We denote by $\mathcal{G}_{\mathcal{U}}^{(1)}$ the sub-group of the elements of $\mathcal{G}_{\mathcal{U}}$ which are trivial on $X(p)(w)$, therefore $\mathcal{G}_{\mathcal{U}} / \mathcal{G}_{\mathcal{U}}^{(1)} \cong G=: G_{1}$. We can choose the basis $e_{0}, e_{1}$ such that for all $\sigma \in \mathcal{G}_{\mathcal{U}}^{(1)}$ we have $\sigma\left(e_{1}\right)=\chi(\sigma) e_{1}$ and $\sigma\left(\operatorname{dlog}_{\mathcal{U}}\left(e_{0}\right)\right)=\operatorname{dlog}_{\mathcal{U}}\left(e_{0}\right)$.

Let $X, Y$ denote the basis of $T^{\vee} \otimes \widehat{\bar{R}}_{\mathcal{U}}$ which is $\widehat{\bar{R}}_{\mathcal{U}}$-dual to $e_{0}, e_{1}$ respectively (i.e., $X\left(e_{1}\right)=$ $Y\left(e_{0}\right)=0$ and $\left.X\left(e_{0}\right)=Y\left(e_{1}\right)=1\right)$. Then the (right) action of $\mathcal{G}_{\mathcal{U}}^{(1)}$ on $X, Y$ is given by:

$$
X \mid \sigma=X \text { and } Y \mid \sigma=\chi(\sigma) Y+\xi(\sigma)^{\sigma^{-1}} X \text { for } \sigma \in \mathcal{G}_{\mathcal{U}}^{(1)}
$$

where $\xi: \mathcal{G}_{\mathcal{U}_{Y}} \longrightarrow \widehat{\bar{R}}_{\mathcal{U}}$ is defined by $\xi(\sigma) e_{1}=\sigma\left(e_{0}\right)-e_{0}$ and if $u \in \widehat{\bar{R}}_{\mathcal{U}}$ and $\alpha \in \mathcal{G}_{\mathcal{U}}^{(1)}$ we denote by $u^{\alpha}$ the natural action of $\alpha$ on $u$. Moreover, as $\mathcal{G}_{\mathcal{U}}^{(1)}$ acts trivially on $X$ we have a natural action of the group $G$ on it given by: for $\sigma \in G, X \mid \sigma=\tau(\sigma) X$ (see remark 4.3 below).

Remark 4.3. In fact we may regard $X$ as an $\widehat{\bar{R}}_{\mathcal{U}}$-linear map $X: F^{0} \longrightarrow \widehat{\bar{R}}_{\mathcal{U}}$, or by restricting it to $\mathcal{F}^{\prime}\left(\bar{R}_{\mathcal{U}}, \bar{N}_{\mathcal{U}}\right)$, as a morphism of $S_{\mathfrak{X}(p)(w)}\left(\bar{R}_{\mathcal{U}}, \bar{N}_{\mathcal{U}}\right)$-torsors

$$
X: \mathcal{F}^{\prime}\left(\bar{R}_{\mathcal{U}}, \bar{N}_{\mathcal{U}}\right) \longrightarrow\left(\widehat{\bar{R}}_{\mathcal{U}}\right)^{(1)},
$$

i.e., an element of $\Omega_{\mathfrak{X}(w)}^{-1}\left(\bar{R}_{\mathcal{U}}, \bar{N}_{\mathcal{U}}\right)$. Moreover, let us recall that $k$ is attached to a pair $(s, i)$ with $s \in K$ (satisfying $v(s)>\frac{p-2}{p-1}$ ) and $0 \leq i \leq p-2$ an integer. Then for $m \in \mathbb{Z}$ we have: $X^{k-m}:=\left(X^{s-i} \cdot X^{i}\right) \cdot X^{-m}:=\exp ((s-i) \log (X)) \cdot X^{i-m}$ is a generator of $\Omega_{\mathfrak{X}(w)}^{-k+m}\left(\bar{R}_{\mathcal{U}}, \bar{N}_{\mathcal{U}}\right)$.

Lemma 4.4. We have

$$
\operatorname{fil}_{m}\left(\mathbb{A}_{k}\right)\left(\bar{R}_{\mathcal{U}}, \bar{N}_{\mathcal{U}}\right)=\sum_{i=0}^{m} \widehat{\bar{R}}_{\mathcal{U}} X^{k}\left(X^{-1} Y\right)^{i},
$$

In particular $\operatorname{fil}_{m}\left(\mathbb{A}_{k}\right)\left(\bar{R}_{\mathcal{U}}, \bar{N}_{\mathcal{U}}\right)$ is a free $\widehat{\bar{R}}_{\mathcal{U}}$-module of rank $m+1$.

Proof. We notice that $\operatorname{Sym}^{m}\left(T^{\vee}\right) \otimes \widehat{\bar{R}}_{\mathcal{U}}=\sum_{i=0}^{m} \widehat{\bar{R}}_{\mathcal{U}} X^{m-i} Y^{i}$ while $\Omega_{\mathfrak{X}(w)}^{-k+m}\left(\bar{R}_{\mathcal{U}}, \bar{N}_{\mathcal{U}}\right)$ is a free $\widehat{\bar{R}}_{\mathcal{U}^{-}}$ module with basis $X^{k-m}$. 
Let us define the morphism $\phi_{m}: \mathrm{fil}_{m}\left(\mathbb{A}_{k}\right) \longrightarrow \mathrm{fil}_{m+1}\left(\mathbb{A}_{k}\right)$ as the composition

$$
\begin{gathered}
\operatorname{fil}_{m}\left(\mathbb{A}_{k}\right)=\operatorname{Sym}^{m}\left(\mathcal{T}^{\vee}\right) \otimes \Omega_{\mathfrak{X}(w)}^{-k+m}= \\
=\operatorname{Sym}^{m}\left(\mathcal{T}^{\vee}\right) \otimes \Omega_{\mathfrak{X}(w)}^{-k+m+1} \otimes \Omega_{\mathfrak{X}(w)}^{-1} \stackrel{\operatorname{dlog}^{\vee}}{\longrightarrow} \operatorname{Sym}^{m+1}\left(\mathcal{T}^{\vee}\right) \otimes \Omega_{\mathfrak{X}(w)}^{-k+m+1}=\operatorname{fil}_{m+1}\left(\mathbb{A}_{k}\right) .
\end{gathered}
$$

Definition 4.5. We set:

$$
\mathbb{A}_{k, i}:=\lim _{\rightarrow, m}\left(\operatorname{fil}_{m}\left(\mathbb{A}_{k}\right) \otimes_{\mathbb{Z}_{p}} \mathbb{Z} / p^{i} \mathbb{Z}, \phi_{m}\right) .
$$

The sheaf $\mathbb{A}_{k, i}$ thus defined is a locally free sheaf of $\mathcal{O}_{\mathfrak{X}(w)} / p^{i} \mathcal{O}_{\mathfrak{X}(w)}$-modules on $\mathfrak{X}(w)$ and we denote by $\mathbb{A}_{k}$ the continuous sheaf of $\widehat{\mathcal{O}}_{\mathfrak{X}(w)}$-modules $\left\{\mathbb{A}_{k, i}\right\}_{i \geq 0}$. We also set

$$
\mathbb{D}_{k, i}:=\operatorname{Hom}_{\mathcal{O}_{\mathfrak{X}(w)} / p^{i} \mathcal{O}_{\mathfrak{X}(w)}}\left(\mathbb{A}_{k, i}, \mathcal{O}_{\mathfrak{X}(w)} / p^{i} \mathcal{O}_{\mathfrak{X}(w)}\right) .
$$

and

$$
\mathbb{D}_{k, i}^{m}:=\operatorname{Ker}\left(\mathbb{D}_{k, i} \longrightarrow \operatorname{Hom}_{\mathcal{O}_{\mathfrak{X}(w)} / p^{i} \mathcal{O}_{\mathfrak{X}(w)}}\left(\operatorname{fil}_{m}\left(\mathbb{A}_{k, i}\right), \mathcal{O}_{\mathfrak{X}(w)} / p^{i} \mathcal{O}_{\mathfrak{X}(w)}\right)\right)
$$

for all $i \geq 0, m \geq 0$, and denote $\mathbb{D}_{k, i}:=\mathbb{D}_{k, i}^{-1}$.

We have the following simple properties of these sheaves.

Lemma 4.6. a) For each $j \geq 0$ we have $\mathbb{A}_{k, j+1} \otimes_{\mathbb{Z}_{p}} \mathbb{Z} / p^{j} \mathbb{Z} \cong \mathbb{A}_{k, j}$.

b) For each $j \geq 0$ we have a natural morphism of $\widehat{\mathcal{O}}_{\mathfrak{X}(w)}$-modules $\mathbb{D}_{k, j+1} \longrightarrow \mathbb{D}_{k, j}$ which give

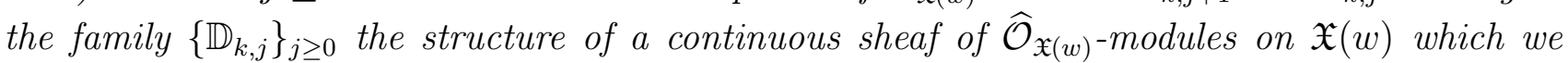
denote $\mathbb{D}_{k}$.

Proof. As $\operatorname{fil}_{m}\left(\mathbb{A}_{k, j}\right)$ is a free $\mathcal{O}_{\mathfrak{X}(w)} / p^{i}\left(\mathcal{O}_{\mathfrak{X}(w)}\right.$-module we have

$$
\operatorname{fil}_{m}\left(\mathbb{A}_{k, j+1}\right) \otimes_{\mathbb{Z}_{p}} \mathbb{Z} / p^{j} \mathbb{Z}=\operatorname{fil}_{m}\left(\mathbb{A}_{k, j+1}\right) \otimes_{\mathcal{O}_{\mathfrak{X}(w)} / p^{j+1} \mathcal{O}_{\mathfrak{X}(w)}} \mathcal{O}_{\mathfrak{X}(w)} / p^{j} \mathcal{O}_{\mathfrak{X}(w)} \cong \operatorname{fil}_{m}\left(\mathbb{A}_{k, j}\right) .
$$

This proves a).

For b) let us notice that we have

$$
\begin{gathered}
\mathbb{D}_{\kappa, j+1}=\operatorname{Hom}_{\mathcal{O}_{\mathfrak{X}(w)} / p^{j+1} \mathcal{O}_{\mathfrak{X}(w)}}\left(\mathbb{A}_{k, j+1}, \mathcal{O}_{\mathfrak{X}(w)} / p^{j+1} \mathcal{O}_{\mathfrak{X}(w)}\right) \stackrel{\circ \phi_{j}}{\longrightarrow} \\
\stackrel{\circ \phi_{j}}{\longrightarrow} \operatorname{Hom}_{\mathcal{O}_{\mathfrak{X}(w)} / p^{j+1} \mathcal{O}_{\mathfrak{X}(w)}}\left(\mathbb{A}_{k, j+1}, \mathcal{O}_{\mathfrak{X}(w)} / p^{j} \mathcal{O}_{\mathfrak{X}(w)}\right) \cong \operatorname{Hom}_{\mathcal{O}_{\mathfrak{X}(w)} / p^{j} \mathcal{O}_{\mathfrak{X}(w)}}\left(\mathbb{A}_{k, j}, \mathcal{O}_{\mathfrak{X}(w)} / p^{j} \mathcal{O}_{\mathfrak{X}(w)}\right)=\mathbb{D}_{k, j}
\end{gathered}
$$

Let us remark that the natural $\widehat{\mathcal{O}}_{\mathfrak{X}(w)}$-linear morphism $\mathbb{D}_{k, j+1} \longrightarrow \mathbb{D}_{k, j}$ of lemma 4.6 induces, for every $m \geq-1$ natural morphisms $\mathbb{D}_{k, j+1}^{m} \longrightarrow \mathbb{D}_{k, j}^{m}$, for all $j \geq 0$, i.e., we obtain a filtration of $\mathbb{D}_{k}$ by $\widehat{\mathcal{O}}_{\mathfrak{X}(w)}$-sub-modules $\left(\mathbb{D}_{k}^{m}:=\left\{\mathbb{D}_{k, j}^{m}\right\}_{j \geq 0}\right)_{m \geq-1}$.

Let $\mathcal{U}:=\left(\operatorname{Spf}\left(R_{\mathcal{U}}\right), N_{\mathcal{U}}\right)$ be a connected small object of $\mathcal{X}(w) \frac{\text { ket }}{K}$ and let us use the notations before lemma 4.4. We have

\section{Lemma 4.7.}

$$
\begin{aligned}
& \text { a) } \mathbb{A}_{k}\left(\widehat{\bar{R}}_{\mathcal{U}}\right)=\left\{\sum_{n=0}^{\infty} a_{n} X^{k-n} Y^{n} \quad \mid \quad a_{n} \in \widehat{\bar{R}}_{\mathcal{U}} \text { with } a_{n} \stackrel{n \rightarrow \infty}{\longrightarrow} 0\right\} \text {. } \\
& \text { b) } \mathbb{D}_{k}\left(\widehat{\bar{R}}_{\mathcal{U}}\right)=\operatorname{Hom}_{\overline{\bar{R}}_{\mathcal{U}}, \text { cont }}\left(A_{k}\left(\widehat{\bar{R}}_{\mathcal{U}}\right),\left(\widehat{\bar{R}}_{\mathcal{U}}\right)\right) \text {. }
\end{aligned}
$$

c) We have

$$
\mathbb{D}_{k, j}^{m}\left(\widehat{\bar{R}}_{\mathcal{U}}\right)=\left\{\mu \in \operatorname{Hom}_{\bar{R}_{\mathcal{U}} / p^{j} \bar{R}_{\mathcal{U}}}\left(\mathbb{A}_{k, j}, \bar{R}_{\mathcal{U}} / p^{j} \bar{R}_{\mathcal{U}}\right) \text { such that }\left.\mu\right|_{\mathrm{fil}_{m} \mathbb{A}_{k, j}}=0\right\} .
$$


Proof. Everything follows from lemma 4.4 and the fact that the sheaves $\operatorname{fil}_{m} \mathbb{A}_{k, j}$ for $m \geq 0$ and $\mathbb{A}_{k, j}$ are free $\mathcal{O}_{\mathfrak{X}(w)} / p^{j} \mathcal{O}_{\mathfrak{X}(w)}$-modules, for all $j \geq 1$.

Corollary 4.8. We have natural isomorphisms as $\widehat{\mathcal{O}}_{\mathfrak{X}(w)}$-modules

$$
\mathbb{A}_{k} \cong \mathcal{A}^{o} \hat{\otimes} \widehat{\mathcal{O}}_{\mathfrak{X}(w)} \text { and } \mathbb{D}_{k} \cong \mathcal{D}_{k}^{o} \hat{\otimes} \widehat{\mathcal{O}}_{\mathfrak{X}(w)} .
$$

Proof. For every $\mathcal{U}:=\left(R_{\mathcal{U}}, N_{\mathcal{U}}\right)$ connected small object of $\mathcal{X}(w) \frac{\text { ket }}{K}$, by lemma 4.7 we have canonical isomorphisms as $\widehat{\bar{R}}_{\mathcal{U}}$-modules, compatible with $\mathcal{G}_{\mathcal{U}}$-actions between the localizations:

$$
\mathbb{A}_{k}\left(\widehat{\bar{R}}_{\mathcal{U}}\right) \cong A_{k}^{o} \hat{\otimes} \widehat{\bar{R}}_{\mathcal{U}} \text { and } \mathbb{D}_{k}\left(\widehat{\bar{R}}_{\mathcal{U}}\right) \cong D_{k}^{o} \hat{\otimes} \widehat{\bar{R}}_{\mathcal{U}}
$$

Now we'd like to determine the graded quotients associated to the filtration $\left(\mathbb{D}_{k}^{m}\right)_{m \geq-1}$ of $\mathbb{D}_{k}$. Let us first remark that if $m \in \mathbb{Z} \subset \mathcal{W}^{*}\left(\mathbb{Q}_{p}\right)$ therefore $\Omega_{\mathfrak{X}(w)}^{m}$ is a free $\widehat{\mathcal{O}}_{\mathfrak{X}(w) \text {-module of rank } 1}$ which satisfies the following properties.

Lemma 4.9. For every $m \geq 0, m \in \mathbb{Z}$ we have an exact sequence of sheaves on $\mathfrak{X}(w)$ :

$$
0 \longrightarrow \operatorname{Sym}^{m}\left(\mathcal{T}^{\vee}\right) \otimes_{\mathbb{Z}_{p}} \Omega_{\mathfrak{X}(w)}^{-1} \stackrel{u}{\longrightarrow} \operatorname{Sym}^{m+1}\left(\mathcal{T}^{\vee}\right) \otimes_{\mathbb{Z}_{p}} \widehat{\mathcal{O}}_{\mathfrak{X}(w)} \longrightarrow \Omega_{\mathfrak{X}(w)}^{m+1}(-m-1) \longrightarrow 0,
$$

where the morphism $u$ is induced by $\mathrm{dlog}^{\vee}$ and the twists are Tate twists.

Proof. Identifying $\mathcal{F}^{0}$ with $\Omega_{\mathfrak{X}(w)}^{1}$ and $\mathcal{F}^{1}$ with $\Omega_{\mathfrak{X}(w)}^{-1}$ (see remark 4.3) we obtain from the HodgeTate sequence $(2)$ the exact sequence of locally free sheaves of $\widehat{\mathcal{O}}_{\mathfrak{X}(w)}$-modules on $\mathfrak{X}(w)$

$$
0 \longrightarrow \Omega_{\mathfrak{X}(w)}^{-1}(1) \longrightarrow \mathcal{T} \otimes \widehat{\mathcal{O}}_{\mathfrak{X}(w)} \longrightarrow \Omega_{\mathfrak{X}(w)}^{1} \longrightarrow 0,
$$

which dualizing gives

$$
0 \longrightarrow \Omega_{\mathfrak{X}(w)}^{-1} \longrightarrow \mathcal{T}^{\vee} \otimes \widehat{\mathcal{O}}_{\mathfrak{X}(w)} \stackrel{u^{\vee}}{\longrightarrow} \Omega_{\mathfrak{X}(w)}^{1}(-1) \longrightarrow 0
$$

We tensor this exact sequence with $\operatorname{Sym}^{m}\left(\mathcal{T}^{\vee}\right) \otimes \widehat{\mathcal{O}}_{\mathfrak{X}(w)}$ (which is a free $\widehat{\mathcal{O}}_{\mathfrak{X}(w)}$-module) and obtain the commutative diagram

$$
\begin{aligned}
& 0 \rightarrow \operatorname{Sym}^{m}\left(\mathcal{T}^{\vee}\right) \otimes \Omega_{\mathfrak{X}(w)}^{-1} \rightarrow \operatorname{Sym}^{m}\left(\mathcal{T}^{\vee}\right) \otimes \mathcal{T}^{\vee} \otimes \widehat{\mathcal{O}}_{\mathfrak{X}(w)} \quad \rightarrow \quad \operatorname{Sym}^{m}\left(\mathcal{T}^{\vee}\right) \otimes \Omega_{\mathfrak{X}(w)}^{1}(-1) \rightarrow 0 \\
& 0 \rightarrow \operatorname{Sym}^{m}\left(\mathcal{T}^{\vee}\right) \otimes \Omega_{\mathfrak{X}(w)}^{-1} \rightarrow \quad \operatorname{Sym}^{m+1}\left(\mathcal{T}^{\vee}\right) \otimes \widehat{\mathcal{O}}_{\mathfrak{X}(w)} \quad \rightarrow \quad \Omega_{\mathfrak{X}(w)}^{m+1}(-m-1) \quad \rightarrow \quad 0
\end{aligned}
$$

Let us first define the arrow: $\psi_{m}: \operatorname{Sym}^{m}\left(\mathcal{T}^{\vee}\right) \otimes \Omega_{\mathfrak{X}(w)}^{1}(-1) \longrightarrow \Omega_{\mathfrak{X}(w)}^{m+1}(-m-1)$.

For $m=0$ the map $\psi_{0}$ is the identity. So let us suppose the map $\psi_{m}$ is defined for $m \geq 0$ such that the diagram is commutative and let us define $\psi_{m+1}$. We first notice that we have a natural map

$$
u^{\vee} \otimes \psi_{m}: \mathcal{T}^{\vee} \otimes \operatorname{Sym}^{m}\left(\mathcal{T}^{\vee}\right) \otimes \Omega_{\mathfrak{X}(w)}^{1}(-1) \longrightarrow \Omega_{\mathfrak{X}(w)}^{m+1}(-m-1) .
$$

Moreover as $\Omega_{\mathfrak{X}(w)}^{1}$ is locally free of rank $1, \Omega_{\mathfrak{X}(w)}^{m+1}=\operatorname{Sym}_{\widehat{\mathcal{O}}_{\mathfrak{X}(w)}^{m+1}}^{m}\left(\Omega_{\mathfrak{X}(w)}^{1}\right)$ and therefore $u^{\vee} \otimes \phi_{m}$ factors uniquely as $\psi_{m}: \operatorname{Sym}^{m}\left(\mathcal{T}^{\vee}\right) \otimes \Omega_{\mathfrak{X}(w)}^{1}(-1) \longrightarrow \Omega_{\mathfrak{X}(w)}^{m+1}(-m-1)$, and the respective diagram is commutative. Now analyzing the localizations at small affines it follows that the bottom sequence is exact which proves the lemma. 
Corollary 4.10. We have

a) $\operatorname{fil}_{m+1}\left(\mathbb{A}_{k}\right) / \operatorname{fil}_{m}\left(\mathbb{A}_{k}\right) \cong \Omega_{\mathfrak{X}(w)}^{-k+2 m+2}(-m-1)$.

b) $\mathbb{D}_{k}^{m} / \mathbb{D}_{k}^{m+1} \cong \Omega_{\mathfrak{X}(w)}^{k-2 m-2}(m+1)$.

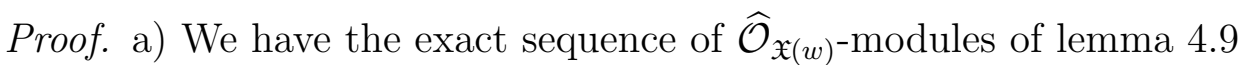

$$
0 \longrightarrow \operatorname{Sym}^{m}\left(\mathcal{T}^{\vee}\right) \otimes_{\mathbb{Z}_{p}} \Omega_{\mathfrak{X}(w)}^{-1} \stackrel{u}{\longrightarrow} \operatorname{Sym}^{m+1}\left(\mathcal{T}^{\vee}\right) \otimes_{\mathbb{Z}_{p}} \widehat{\mathcal{O}}_{\mathfrak{X}(w)} \longrightarrow \Omega_{\mathfrak{X}(w)}^{m+1}(-m-1) \longrightarrow 0
$$

Now we tensor over $\widehat{\mathcal{O}}_{\mathfrak{X}(w)}$ with $\Omega_{\mathfrak{X}(w)}^{-k+m+1}$ and obtain the exact sequence

$$
0 \longrightarrow \operatorname{Sym}^{m}\left(\mathcal{T}^{\vee}\right) \otimes_{\mathbb{Z}_{p}} \Omega_{\mathfrak{X}(w)}^{-k+m} \longrightarrow \operatorname{Sym}^{m+1}\left(\mathcal{T}^{\vee}\right) \otimes_{\mathbb{Z}_{p}} \Omega_{\mathfrak{X}(w)}^{-k+m+1} \longrightarrow \Omega_{\mathfrak{X}(w)}^{-k+2 m+2}(-m-1) \longrightarrow 0,
$$

which proves a).

b) We dualize the exact sequence above and obtain

$$
\mathbb{D}_{k}^{m} / \mathbb{D}_{k}^{m+1}=\operatorname{Hom}_{\widehat{\mathcal{O}}_{\mathfrak{X}(w)}}\left(\operatorname{fil}_{m+1}\left(\mathbb{A}_{k}\right) / \mathrm{fil}_{m}\left(\mathbb{A}_{k}\right), \widehat{\mathcal{O}}_{\mathfrak{X}(w)}\right) \cong \Omega_{\mathfrak{X}(w)}^{k-2 m-2}(m+1)
$$

In particular, for $m=-1$ we have

$$
\mathbb{D}_{k} / \mathbb{D}_{k}^{0} \cong \Omega_{\mathfrak{X}(w)}^{k}
$$

\subsection{The map $d \log ^{\vee}, k$.}

We start by fixing a $K$-valued weight $k$ as in the previous section. In particular $w$ is accessible and the associated $r$ is equal to 1 . Let $w$ be adapted to $k$. We have explained in section 3.3 how to construct a continuous sheaf $\mathcal{A}_{k}^{o}=\left(\mathcal{A}_{k, n}^{o}\right)_{n \in \mathbb{N}}$ on Faltings' site $\mathfrak{X}(w)$ associated to the continuous representation of $A_{k}^{o}=\left(A_{k}^{o} / \underline{m}^{n} A_{k}^{o}\right)_{n \in \mathbb{N}}$ of the Kummer étale fundamental group $\mathcal{G}$ of $X(N, p)$. We denoted by $\mathbb{A}_{k}:=\mathcal{A}_{k}^{o} \hat{\otimes} \widehat{\mathcal{O}}_{\mathfrak{X}(w)}$. Similarly we have the sheaves $\mathbb{D}_{k}=\left(\mathbb{D}_{k, n}\right)_{n \in \mathbb{N}}$. By construction the sheaf $\mathbb{D}_{k, n}$ is a quotient of $\mathfrak{H o m}_{\mathcal{O}_{K}}\left(\mathbb{A}_{k, n}, \mathcal{O}_{K} / \underline{m}^{m}\right)$.

We write $\mathcal{T}_{0}$ for the continuous sheaf on $\mathfrak{X}(w)$ associated to the $\mathcal{G}$-representation $T_{0}$. Then we have an inclusion of sheaves

$$
\mathcal{A}_{k, n}^{o} \subset \mathfrak{H o m}_{\mathbb{Z}_{p}^{*}}\left(\mathcal{T}_{0},\left(\mathcal{O}_{K} / \underline{m}^{n}\right)^{(k)}\right)
$$

on $\mathfrak{X}(w)$, which for every $r$ and $n \in \mathbb{N}$ provides a map of sheaves of $\mathcal{O}_{\mathfrak{X}\left(p^{r}\right)(w)} \otimes \mathcal{O}_{K} / \underline{m}^{n}$-modules

$$
\beta_{n}^{(r)}: j_{r}^{*}\left(\mathcal{A}_{k, n}^{o}\right) \otimes_{\mathcal{O}_{K}}\left(\mathcal{O}_{\mathfrak{X}\left(p^{r}\right)(w)} / p^{n} \mathcal{O}_{\mathfrak{X}\left(p^{r}\right)(w)}\right) \longrightarrow \mathfrak{H o m}_{\mathbb{Z}_{p}^{*}}\left(j_{r}^{*}\left(\mathcal{T}_{0}\right),\left(\mathcal{O}_{\mathfrak{X}\left(p^{r}\right)(w)} \otimes \mathcal{O}_{K} / \underline{m}^{n}\right)^{(-k)}\right) .
$$

These maps are compatible for varying $n$ and define a map of continuous sheaves

$$
\beta^{(r)}: j_{r}^{*}\left(\mathcal{A}_{k}^{o}\right) \widehat{\otimes}_{\mathcal{O}_{K}} \widehat{\mathcal{O}}_{\mathfrak{X}\left(p^{r}\right)(w)} \longrightarrow \mathfrak{H o m}_{\mathbb{Z}_{p}^{*}}\left(j_{r}^{*}\left(\mathcal{T}_{0}\right), \widehat{\mathcal{O}}_{\mathfrak{X}\left(p^{r}\right)(w)}\right) \text {. }
$$

This map has the following properties (see [AIS2] proposition 4.8):

(1) The map $\beta^{(r)}$ is injective and $G_{r}$-invariant.

(2) The map d $\log ^{\vee}, k$ is $G_{r}$-invariant and factors via $\beta^{(r)}$. 
For the rest of this section we assume $r=1$. From the discussion above it follows that dlog ${ }^{\vee}, k$ defined in section 4.1 induces a $G_{1}$-invariant morphism of $\widehat{\mathcal{O}}_{\mathfrak{X}(p)(w)}$-modules

$$
\mathcal{M}_{k}^{(1)}(w) \longrightarrow j_{1}^{*}\left(\mathcal{A}_{k}^{o}\right) \widehat{\otimes}_{\mathcal{O}_{K}} \widehat{\mathcal{O}}_{\mathfrak{X}(p)(w)}
$$

Applying the functor $\mathfrak{H o m}_{\mathcal{O}_{\mathfrak{X}(p)(w)}}\left(-, \mathcal{O}_{\mathfrak{X}(p)(w)}\right)$ and using the identification

$$
\mathfrak{H o m}_{\mathcal{O}_{\mathfrak{X}(p)(w)}}\left(\mathcal{M}_{k}^{(1)}(w), \mathcal{O}_{\mathfrak{X}(p)(w)}\right) \cong \mathcal{M}_{-k}^{(1)}(w),
$$

we get an induced $G_{1}$-invariant morphism $\mathcal{O}_{K}$-modules

$$
\delta: \mathfrak{H o m}_{\mathcal{O}_{K}}\left(j_{1}^{*}\left(\mathcal{A}_{k}^{o}\right), \mathcal{O}_{K}\right) \longrightarrow \mathcal{M}_{-k}^{(1)}(w)
$$

Then, for every $n \in \mathbb{N}$ there exists $m \geq n$ such that the map

$$
\mathfrak{H} \mathfrak{m}_{\mathcal{O}_{K}}\left(j_{1}^{*}\left(\mathcal{A}_{k, m}^{o}(w)\right), \mathcal{O}_{K} / \underline{m}^{m}\right) \longrightarrow \mathfrak{H} o m_{\mathcal{O}_{\mathfrak{X}(p)(w)}}\left(\mathcal{M}_{k}^{(1)}(w),\left(\mathcal{O}_{\mathfrak{X}(p)(w)} \otimes \mathcal{O}_{K} / \underline{m}^{n}\right)^{(-k)}\right)
$$

induced by $\delta$, factors via $j_{1}^{*}\left(\mathcal{D}_{k, m}^{o}\right)$.

It follows that we get a map of continuous sheaves of $\widehat{\mathcal{O}}_{\mathfrak{X}(w)}$-modules

$$
\mathbb{D}_{k} \longrightarrow\left(j_{1, *}\left(j_{1}^{*}\left(\mathcal{D}_{k}^{o}\right)\right)\right)^{G_{1}} \hat{\otimes} \widehat{\mathcal{O}}_{\mathfrak{X}(w)} \longrightarrow\left(j_{1, *}\left(\mathcal{M}_{-k}^{(1)}(w)\right)\right)^{G_{1}}=\Omega_{\mathfrak{X}(w)}^{\dagger, k}
$$

Passing to ind-sheaves (i.e., inverting $p$ ) we obtain a map

$$
\delta_{k}^{\vee}(w): D_{k, K}=\mathbb{D}_{k}[1 / p] \longrightarrow \omega_{\mathfrak{X}(w)}^{\dagger, k} \cong \omega_{w}^{\dagger, k} \hat{\otimes}_{\widehat{\mathcal{O}}_{\mathcal{X}(w)}} \widehat{\mathcal{O}}_{\mathfrak{X}(w)}
$$

In the next section we will calculate the cohomology of the ind-continuous sheaves $\omega_{\mathfrak{X}(w)}^{\dagger, k} \cong$ $\omega_{w}^{\dagger, k} \hat{\otimes}_{\widehat{\mathcal{O}}_{\mathcal{X}(w)}} \widehat{\mathcal{O}}_{\mathfrak{X}(w)}$.

\subsection{The cohomology of the sheaves $\omega_{\mathfrak{X}(w)}^{\dagger, k}$}

Let $\iota: Z \longrightarrow X(w)$ be a morphism in $\mathcal{X}(w) \frac{\text { fket }}{K}$. Let $\mathfrak{Z}:=\mathfrak{X}(w)_{/(\mathcal{X}(w), Z)}$ the associated induced site and $j:=j_{(\mathcal{X}(w), Z)}: \mathfrak{X}(w) \longrightarrow \mathfrak{Z}$ the $\operatorname{map} j(\mathcal{U}, W):=\left(\mathcal{U}, Z \times_{X(w)} W, \operatorname{pr}_{1}\right)$; see 2.3. It induces a morphism of topoi. For $i \geq 0$ we shall calculate $\mathrm{H}^{i}\left(\mathcal{Z}, j^{*}\left(\omega_{\mathfrak{X}(w)}^{\dagger, k}\right)\right)$. For $\iota=$ id we get in particular the calculation of $\mathrm{H}^{i}\left(\mathfrak{X}(w), \omega_{\mathfrak{X}(w)}^{\dagger, k}\right)$.

Theorem 4.11. [AIS2]. We have isomorphisms as $G_{K}$-modules

a) $\mathrm{H}^{0}\left(\mathfrak{Z}, j^{*}\left(\omega_{\mathfrak{X}(w)}^{\dagger, k}(1)\right)\right) \cong \mathrm{H}^{0}\left(Z, \iota^{*}\left(\omega_{w}^{\dagger, k}\right)\right) \hat{\otimes}_{K} \mathbb{C}_{p}(1)$;

b) $\mathrm{H}^{1}\left(\mathfrak{Z}, j^{*}\left(\omega_{\mathfrak{X}(w)}^{\dagger, k}(1)\right)\right) \cong \mathrm{H}^{0}\left(Z, \iota^{*}\left(\omega_{w}^{\dagger, k+2}\right)\right) \hat{\otimes}_{K} \mathbb{C}_{p}$;

c) $\mathrm{H}^{i}\left(\mathfrak{Z}, j^{*}\left(\omega_{\mathfrak{X}(w)}^{\dagger, k}(1)\right)\right)=0$ for $i \geq 2$.

Proof. As $R^{i} j_{*}=0$ for all $i \geq 1$ by [AIS2] corollary 2.6 we have $\mathrm{H}^{i}\left(\mathfrak{Z}, j^{*}\left(\omega_{\mathfrak{X}(w)}^{\dagger, k}(1)\right)\right) \cong$ $\mathrm{H}^{1}\left(\mathfrak{X}(w), j_{*}\left(j^{*}\left(\omega_{\mathfrak{X}(w)}^{\dagger, k}(1)\right)\right)\right)$. Set $\mathcal{F}:=j_{*}\left(j^{*}\left(\omega_{\mathfrak{X}(w)}^{\dagger, k}(1)\right)\right)$. Recall that $\omega_{\mathfrak{X}(w)}^{\dagger, k}$ is isomorphic to $\omega_{w}^{\dagger, k} \hat{\otimes}_{\widehat{\mathcal{O}}_{\mathcal{X}(w)}} \widehat{\mathcal{O}}_{\mathfrak{X}(w)}$. Thus, $j^{*}\left(\omega_{\mathfrak{X}(w)}^{\dagger, k}(1)\right) \cong \omega_{w}^{\dagger, k} \hat{\otimes}_{\widehat{\mathcal{O}}_{\mathcal{X}(w)}} \widehat{\mathcal{O}}_{\mathfrak{Z}}(1)$ as $j^{*}\left(\widehat{\mathcal{O}}_{\mathfrak{X}(w)}\right) \cong \widehat{\mathcal{O}}_{\mathfrak{Z}}$ and $\mathcal{F} \cong \omega_{w}^{\dagger, k} \hat{\otimes}_{\widehat{\mathcal{O}}_{\mathcal{X}(w)}} j_{*}\left(\widehat{\mathcal{O}}_{\mathfrak{Z}}\right)(1)$. The natural map $\widehat{\mathcal{O}}_{\mathfrak{X}(w)} \hat{\otimes}_{\widehat{\mathcal{O}}_{\mathcal{X}(w)}} \iota_{*}\left(\widehat{\mathcal{O}}_{Z}\right) \longrightarrow j_{*}\left(\widehat{\mathcal{O}}_{\mathfrak{Z}}\right)\left[p^{-1}\right]$ is an isomorphism. Hence, $\mathcal{F} \cong \omega_{w}^{\dagger, k} \hat{\otimes}_{\widehat{\mathcal{O}}_{\mathcal{X}(w)}} \iota_{*}\left(\widehat{\mathcal{O}}_{\mathcal{Z}}\right)(1)$ is a locally free $\left(\widehat{\mathcal{O}}_{\mathfrak{X}(w)}[1 / p]\right.$-module. 
To prove the theorem we will first calculate the sheaves $R^{b} v_{\mathfrak{X}(w), *}(\mathcal{F})$ using lemma 2.1 and then we'll use the Leray spectral sequence:

$$
\mathrm{H}^{a}\left(\mathcal{X}(w)^{\mathrm{ket}}, R^{b} v_{\mathfrak{X}(w), *}(\mathcal{F})\right) \Longrightarrow \mathrm{H}^{i}(\mathfrak{X}(w), \mathcal{F}) .
$$

We compute the Galois cohomology of the localization of the ind-continuous sheaf $\mathcal{F}$

$$
\mathcal{F}\left(\bar{R}_{\mathcal{U}}, \bar{N}_{\mathcal{U}}\right)=\omega_{w}^{\dagger, k}(\mathcal{U}) \hat{\otimes}_{R_{\mathcal{U}, K} \iota_{*}}\left(\mathcal{O}_{Z}\right)\left(\mathcal{U}_{K}\right) \hat{\otimes}_{R_{\mathcal{U}}} \widehat{\bar{R}}_{\mathcal{U}}(1) .
$$

for $\mathcal{U}=\left(\operatorname{Spf}\left(R_{\mathcal{U}}, N_{\mathcal{U}}\right)\right.$ a small affine open of $\mathcal{X}(w)^{\mathrm{ket}}$. Using that $\omega_{\mathfrak{X}(w)}^{\dagger, k}$ is a locally free $\left(\widehat{\mathcal{O}}_{\mathfrak{X}(w)} \hat{\otimes} A\right)[1 / p]$-module of rank 1, it follows from the main result of $[\mathrm{Fa}]$ that $\mathrm{H}^{0}\left(\mathcal{G}_{\mathcal{U}}, \widehat{\bar{R}}_{\mathcal{U}, K}\right)=$ $R_{\mathcal{U}, K} \hat{\otimes}_{K} \mathbb{C}_{p}$ so that

$$
\mathrm{H}^{0}\left(\mathcal{G}_{\mathcal{U}}, \mathcal{F}\left(\bar{R}_{\mathcal{U}}, \bar{N}_{\mathcal{U}}\right)\right)=\omega_{w}^{\dagger, k}(\mathcal{U}) \hat{\otimes}_{R_{\mathcal{U}, K} \iota_{*}}\left(\mathcal{O}_{Z}\right)\left(\mathcal{U}_{K}\right) \hat{\otimes}_{K} \mathbb{C}_{p}(1) .
$$

Moreover $\mathrm{H}^{1}\left(\mathcal{G}_{\mathcal{U}}, \widehat{\bar{R}}_{\mathcal{U}, K}\right) \cong \Omega_{\mathcal{U}_{K} / K}^{1} \hat{\otimes}_{K} \mathbb{C}_{p}(-1)$ so that

$$
\mathrm{H}^{1}\left(\mathcal{G}_{\mathcal{U}}, \mathcal{F}\left(\bar{R}_{\mathcal{U}}, \bar{N}_{\mathcal{U}}\right)\right) \cong\left(\Omega_{\mathcal{U}_{K} / K}^{1} \hat{\otimes}_{K} \mathbb{C}_{p}(-1)\right) \hat{\otimes}_{R_{\mathcal{U}, K} \iota_{*}}\left(\mathcal{O}_{Z}\right)\left(\mathcal{U}_{K}\right) \hat{\otimes}_{K} \omega_{w}^{\dagger, k}(\mathcal{U})(1)
$$

The Kodaira-Spencer isomorphism gives $\Omega_{\mathcal{U}_{K} / K}^{1} \hat{\otimes}_{K} B_{K} \cong \omega_{\mathcal{U}_{K}}^{\otimes 2} / \mathcal{U}_{K} \hat{\otimes}_{K} B_{K} \cong \omega_{w}^{\dagger, 2}(\mathcal{U})$. Therefore we obtain

$$
\mathrm{H}^{1}\left(\mathcal{G}_{\mathcal{U}}, \mathcal{F}\left(\bar{R}_{\mathcal{U}}, \bar{N}_{\mathcal{U}}\right)\right) \cong \omega_{w}^{\dagger, k+2}(\mathcal{U}) \hat{\otimes}_{R_{\mathcal{U}, K} \iota_{*}}\left(\mathcal{O}_{Z}\right)\left(\mathcal{U}_{K}\right) \hat{\otimes}_{K} \mathbb{C}_{p}
$$

Finally $\mathrm{H}^{i}\left(\mathcal{G}_{\mathcal{U}}, \mathcal{F}\left(\bar{R}_{\mathcal{U}}, \bar{N}_{\mathcal{U}}\right)\right)=0$ for $i \geq 2$ because $\mathcal{G}_{\mathcal{U}}$ has cohomological dimension 1 . It follows that we have

$$
R^{0} v_{\mathfrak{X}(w), *} \mathcal{F} \cong \omega_{w}^{\dagger, k} \hat{\otimes}_{\mathcal{R}_{\mathcal{U}, K} \iota_{*}}\left(\mathcal{O}_{Z}\right)\left(\mathcal{U}_{K}\right) \hat{\otimes}_{K} \mathbb{C}_{p}(1),
$$

where the isomorphism is as sheaves on $\mathcal{X}(w)^{\mathrm{ket}}$. Similarly we have

$$
R^{1} v_{\mathfrak{X}(w), *} \mathcal{F}=\omega_{w}^{\dagger, k+2} \hat{\otimes}_{R_{\mathcal{U}, K} \iota_{*}}\left(\mathcal{O}_{Z}\right)\left(\mathcal{U}_{K}\right) \hat{\otimes}_{K} \mathbb{C}_{p},
$$

and $R^{b} v_{\mathfrak{X}(w), *} \mathcal{F}=0$ for $b \geq 2$. Now let us observe that $\omega^{\dagger, k} \hat{\otimes}_{R_{\mathcal{U}, K} \iota_{*}}\left(\mathcal{O}_{Z}\right)\left(\mathcal{U}_{K}\right) \hat{\otimes}_{K} \mathbb{C}_{p}(1)$ is a sheaf of $K$-Banach modules on $X(w)$, as it is locally isomorphic to $\iota_{*}\left(\mathcal{O}_{Z}\right) \hat{\otimes} B_{K} \hat{\otimes} \mathbb{C}_{p}$. As $X(w)$ is an affinoid we obtain that

$$
\mathrm{H}^{1}\left(\mathcal{X}(w)^{\mathrm{ket}}, \omega_{w}^{\dagger, k} \hat{\otimes}_{\mathcal{O}_{\mathcal{X}(w)}} \iota_{*}\left(\mathcal{O}_{Z}\right) \hat{\otimes}_{K} \mathbb{C}_{p}(1)\right)=\mathrm{H}^{1}\left(X(w), \omega_{w}^{\dagger, k} \hat{\otimes}_{\mathcal{O}_{\mathcal{X}}(w)} \iota_{*}\left(\mathcal{O}_{Z}\right) \hat{\otimes} \mathbb{C}_{p}(1)\right)=0,
$$

by Kiehl's vanishing theorem. Therefore the Leray spectral sequence gives now the result of the theorem.

\section{The main result}

Let $k \in \mathcal{W}^{*}(K)$ be an accessible weight and $h \in \mathbb{Q}, h \geq 0$ a slope. Let us recall the morphism of continuous sheaves on $\mathfrak{X}(w)$ defined in section 4.3

$$
\delta_{k}^{\vee}(w): D_{k, K}=\mathbb{D}_{k}[1 / p] \longrightarrow \omega_{\mathfrak{X}(w)}^{\dagger, k} \cong \omega_{w}^{\dagger, k} \hat{\otimes}_{\widehat{\mathcal{O}}_{\mathcal{X}(w)}} \widehat{\mathcal{O}}_{\mathfrak{X}(w)} .
$$

It induces a $G_{K}$ and Hecke equivariant morphism

$$
\mathfrak{R}_{k}: \mathrm{H}^{1}\left(\mathfrak{X}(w), \mathbb{D}_{k}(1)\right) \longrightarrow \mathrm{H}^{1}\left(\mathfrak{X}(w), \omega_{w}^{\dagger, k} \hat{\otimes} \widehat{\mathcal{O}}_{\mathfrak{X}(w)}(1)\right) \longrightarrow \mathrm{H}^{0}\left(X(w), \omega_{w}^{\dagger, k+2}\right) \hat{\otimes}_{K} \mathbb{C}_{p} .
$$

Notice that for $w$ close enough to 0 the Hecke module $\mathrm{H}^{0}\left(X(w), \omega_{w}^{\dagger, k+2}\right)$ is identified with Coleman's notion of overconvergent form of weight $k+2$ thanks to [AIS1] section 5.1 and theorem 5.1. In this chapter we prove the main result of this note, i.e., the following theorem 
Theorem 5.1. a) The $\mathbb{C}_{p}$-vector space $\mathrm{H}^{1}\left(\mathfrak{X}(w), \mathbb{D}_{k}(1)\right)$ has a natural slope $h$-decomposition, and

b) $\mathfrak{R}_{k}$ induces a $G_{K}$ and Hecke equivariant isomorphism

$$
\mathfrak{R}_{k}^{(h)}: \mathrm{H}^{1}\left(\mathfrak{X}(w), \mathbb{D}_{k}(1)\right)^{(h)} \cong \mathrm{H}^{0}\left(X(w), \omega_{w}^{\dagger, k+2}\right)^{(h)} \otimes_{K} \mathbb{C}_{p}
$$

The proof of this theorem will occupy the rest of the chapter.

\subsection{The cohomology of the quotients $\mathbb{D}_{k, K}^{m} / \mathbb{D}_{k, K}^{m+n}$}

We start by fixing an accessible weight $k \in \mathcal{W}^{*}(K)$ and $w \in \mathbb{Q}$ such that $0<w<p /(p+1)$. In this section we propose to calculate the cohomology groups $\mathrm{H}^{i}\left(\mathfrak{X}(w), \mathbb{D}_{k, K}^{m} / \mathbb{D}_{k, K}^{m+n}\right)$ for all $m \geq$ $-1, n \geq 1$. Here $\mathfrak{X}(w)$ is Faltings' site associated to the pair $(\mathcal{X}(w), X(w))$ and $\mathbb{D}_{k, K}:=\mathbb{D}_{k} \otimes K$ is the ind-continuous sheaf of $\widehat{\mathcal{O}}_{\mathfrak{X}(w)} \otimes K$-modules on $\mathfrak{X}(w)$ defines in section 4.3. It is endowed with a natural filtration $\left\{\mathbb{D}_{k, K}^{m}:=\mathbb{D}_{k}^{m} \otimes K\right\}_{m \geq-1}$.

Let us recall how to calculate these cohomology groups. Let $\mathcal{F}$ be an ind-continuous sheaf on $\mathfrak{X}(w)$. We have the Leray spectral sequence

$$
\mathrm{H}^{a}\left(\mathcal{X}(w)^{\mathrm{ket}}, R^{b} v_{\mathfrak{X}(w), *}(\mathcal{F})\right) \Longrightarrow \mathrm{H}^{a+b}(\mathfrak{X}(w), \mathcal{F})
$$

Moreover, thanks to 2.1 for all $b \geq 0$ the sheaves $R^{b} v_{\mathfrak{X}(w), *}(\mathcal{F})$ can be computed using the Galois cohomology of the localizations of $\mathcal{F}$ at small affines, i.e., it is the sheaf on $\mathcal{X}(w)^{\text {ket }}$ associated to the presheaf

$$
\mathcal{U}:=\left(\operatorname{Spf}\left(R_{\mathcal{U}}\right), N_{\mathcal{U}}\right) \longrightarrow \mathrm{H}^{b}\left(\mathcal{G}, \mathcal{F}\left(\bar{R}_{\mathcal{U}}, \bar{N}_{\mathcal{U}}\right)\right)
$$

where $\mathcal{G}:=\operatorname{Gal}\left(\bar{R}_{K} / R_{\mathcal{U}, \bar{K}}\right)$ is the Kummer-étale geometric fundamental group of $\mathcal{U}_{K}$ for the chosen geometric generic point.

We now fix $m \geq-1, s \geq 1$ and we have.

Proposition 5.2. i) $R^{0} v_{\mathfrak{X}(w), *}\left(\mathbb{D}_{k}^{m} / \mathbb{D}_{k}^{m+s}\right)=\Omega_{w}^{k-2 m-2 s+2} \otimes_{\mathcal{O}_{K}} \mathcal{O}_{\mathbb{C}_{p}}(m+s-1)$.

ii) $R^{1} v_{\mathfrak{X}(w), *}\left(\mathbb{D}_{k, K}^{m} / \mathbb{D}_{k, K}^{m+s}\right) \cong \omega_{w}^{\dagger, k-2 m} \otimes_{K} \mathbb{C}_{p}(m)$.

iii) $\left.R^{b} v_{\mathfrak{X}(w), *}\left(\mathbb{D}_{k, K}^{m} / \mathbb{D}_{k, K}^{m+s}\right)\right)=0$ for $b \geq 2$.

Proof. Let us first notice that iii) is a consequence of the fact that $\mathcal{G}$ has cohomological dimension 1.

Let us fix $\mathcal{U}:=\left(\operatorname{Spf}\left(R_{\mathcal{U}}\right), N_{\mathcal{U}}\right)$ a small affine of $\mathcal{X}(w)^{\text {ket }}$ and let $e_{0}, e_{1}$ be the $\widehat{\bar{R}}_{\mathcal{U}}$-basis of $T \otimes \widehat{\bar{R}}_{\mathcal{U}}$ defined there with $X, Y$ the corresponding $\widehat{\bar{R}}_{\mathcal{U}}$-dual basis. We recall that we denoted by $\Delta:=\mathcal{G}^{(1)}$ the open subgroup of the fundamental group $\mathcal{G}$ of automorphisms which fix the $\log$ affinoid $\mathcal{V}_{K}$ where $\mathcal{V}:=\mathcal{U} \times_{\mathcal{X}(w)} \mathcal{X}(p)(w)$, i.e., $\Delta$ is the kernel of the map $\mathcal{G} \longrightarrow G=$ $\operatorname{Gal}(X(p)(w) / X(w)) \cong \mathbb{F}_{p}^{\times}$. Let us also recall that the (right) action of $\Delta$ on $X, Y$ is given by

$$
X \mid \sigma=X \text { and } Y \mid \sigma=\chi(\sigma) Y+\xi(\sigma)^{\sigma^{-1}} X \text { for all } \sigma \in \Delta .
$$

With these notations, $\mathbb{D}_{k, K}^{m}\left(\bar{R}_{\mathcal{U}}\right)=\left\{\mu \in \mathbb{D}_{k, K}\right.$ such that $\mu\left(X^{k-n} Y^{n}\right)=0$ for all $\left.0 \leq n \leq m\right\}$.

Let us first remark that the localization of the isomorphism of corollary 4.10 at $\mathcal{U}$ can be described explicitly as follows. We have a natural map, $\mathcal{G}$-equivariant,

$$
\mathbb{D}_{k}^{m}\left(\bar{R}_{\mathcal{U}}, \bar{N}_{\mathcal{U}}\right) \longrightarrow \Omega_{\mathfrak{X}}^{k-2 m-2}\left(\bar{R}_{\mathcal{U}}, \bar{N}_{\mathcal{U}}\right)(m+1) \text { defined by } \mu \rightarrow \mu\left(X^{k-m-1} Y^{m+1}\right)
$$


which is surjective and whose kernel is $\mathbb{D}_{k}^{m+1}\left(\bar{R}_{\mathcal{U}}, \bar{N}_{\mathcal{U}}\right)$. Therefore we have an $\widehat{\bar{R}}_{\mathcal{U}}$-linear isomorphism which is $\mathcal{G}$-equivariant (we recall that the action of $\mathcal{G}$ is semi-linear) $\left(\mathbb{D}_{k}^{m} / \mathbb{D}_{k}^{m+1}\right)\left(\bar{R}_{\mathcal{U}}, \bar{N}_{\mathcal{U}}\right) \cong$ $\Omega_{\mathfrak{X}}^{k-2 m-2}\left(\bar{R}_{\mathcal{U}}, \bar{N}_{\mathcal{U}}\right)(m+1)$. Using the main result of $[\mathrm{Fa}]$ we obtain that:

$$
\mathrm{H}^{0}\left(\mathcal{G},\left(\mathbb{D}_{k}^{m} / \mathbb{D}_{k}^{m+1}(1)\right)\left(\bar{R}_{\mathcal{U}}, \bar{N}_{\mathcal{U}}\right)\right) \cong \Omega_{w}^{k-2 m-2}(\mathcal{U}) \hat{\otimes}_{\mathcal{O}_{K}} \mathcal{O}_{\mathbb{C}_{p}}(m+2),
$$

Moreover we have

$$
\begin{gathered}
\mathrm{H}^{1}\left(\mathcal{G},\left(\mathbb{D}_{k, K}^{m} / \mathbb{D}_{k, K}^{m+1}(1)\right)\left(\bar{R}_{\mathcal{U}}, \bar{N}_{\mathcal{U}}\right)\right) \cong \omega_{w}^{\dagger, k-2 m-2}(\mathcal{U}) \otimes_{K} \mathbb{C}_{p}(m+2) \otimes_{\mathbb{C}_{p}} \mathrm{H}^{1}\left(\mathcal{G}_{\bar{R}_{\mathcal{U}}} \otimes K(1)\right) \cong \\
\omega_{w}^{\dagger, k-2 m-2}(\mathcal{U})(m+1) \otimes_{K} \Omega_{\mathcal{U}_{K} / K}^{1} \otimes_{K} \mathbb{C}_{p} \cong \omega_{w}^{\dagger, k-2 m}(\mathcal{U}) \otimes_{K} \mathbb{C}_{p}(m+1) .
\end{gathered}
$$

The last isomorphism is induced by the Kodaira-Spencer isomorphism: $\omega_{w}^{\dagger, 2} \cong \omega_{\mathcal{E}_{K} / \mathcal{U}_{K}}^{2} \cong \Omega_{\mathcal{U}_{K} / K}^{1}$. It follows that the proposition 5.2 is proved for $s=1$.

Let now $s=2$ and let us consider the exact sequence of $\mathcal{G}_{\mathcal{U}}$-modules

$$
(*) \quad 0 \longrightarrow\left(\mathbb{D}_{k}^{i+1} / \mathbb{D}_{k}^{i+2}\right)\left(\bar{R}_{\mathcal{U}}, \bar{N}_{\mathcal{U}}\right) \longrightarrow\left(\mathbb{D}_{k}^{i} / \mathbb{D}_{k}^{i+2}\right)\left(\bar{R}_{\mathcal{U}}, \bar{N}_{\mathcal{U}}\right) \longrightarrow\left(\mathbb{D}_{k}^{i} / \mathbb{D}_{k}^{i+1}\right)\left(\bar{R}_{\mathcal{U}}, \bar{N}_{\mathcal{U}}\right) \longrightarrow 0
$$

As $\mathbb{D}_{k}^{j} / \mathbb{D}_{k}^{j+1} \cong \Omega_{\mathfrak{X}(w)}^{k-2 j-2}(j+1)$, we have that the class of the extension $(*)$ restricted to $\Delta_{\mathcal{U}}$ is a class in

$$
\operatorname{Ext}_{\bar{R}_{\mathcal{U}}, \Delta_{\mathcal{U}}}^{1}\left(\Omega_{\mathfrak{X}(w)}^{k-2 i-2}\left(\bar{R}_{\mathcal{U}}, \bar{N}_{\mathcal{U}}\right)(i+1), \Omega_{\mathfrak{X}(w)}^{\kappa-2 i-4}\left(\bar{R}_{\mathcal{U}}, \bar{N}_{\mathcal{U}}\right)(i+2)\right) \cong \mathrm{H}^{1}\left(\Delta_{\mathcal{U}}, \Omega_{\mathfrak{X}(w)}^{-2}\left(\bar{R}_{\mathcal{U}}\right)(-1)\right)
$$

and we'd like to calculate it. For this let $\mu_{0}, \mu_{1} \in \mathbb{D}_{k}^{i}\left(\bar{R}_{\mathcal{U}}, \bar{N}_{\mathcal{U}}\right)$ be distributions such that

- $\mu_{0} \in \mathbb{D}_{k}^{i+1}\left(\bar{R}_{\mathcal{U}}, \bar{N}_{\mathcal{U}}\right)$ is such that $\mu_{0}\left(X^{k-i-2} Y^{i+2}\right)=1$

and

- $\mu_{1}\left(X^{k-i-2} Y^{i+2}\right)=0$ and $\mu_{1}\left(X^{k-i-1} Y^{i+1}\right)=1$.

It is then easy to see that $\mu_{0}, \mu_{1}$ is an $\widehat{\bar{R}}_{\mathcal{U}}$-basis of $\left(\mathbb{D}_{k}^{i} / \mathbb{D}_{k}^{i+2}\right)\left(\bar{R}_{\mathcal{U}}, \bar{N}_{\mathcal{U}}\right)$ on which $\Delta_{\mathcal{U}}$ acts as follows

$$
\sigma\left(\mu_{0}\right)=\chi(\sigma)^{i+2} \mu_{0} \text { and } \sigma\left(\mu_{1}\right)=\chi(\sigma)^{i+1}\left(\mu_{1}+(i+2) \xi(\sigma) \mu_{0}\right)
$$

In other words the class of $(*)$ seen as an element of $\mathrm{H}^{1}\left(\Delta_{\mathcal{U}}, \Omega_{\mathfrak{X}(w)}^{-2}\left(\bar{R}_{\mathcal{U}}, \bar{N}_{\mathcal{U}}\right)(-1)\right.$ ) is (an integer) $\times \xi$. Therefore the boundary map $\partial$ in the long exact cohomology sequence associated to $(*)$

$$
\mathrm{H}^{0}\left(\Delta,\left(\mathbb{D}_{k}^{i} / \mathbb{D}_{k}^{i+1}\right)\left(\bar{R}_{\mathcal{U}}, \bar{N}_{\mathcal{U}}\right)\right) \stackrel{\partial}{\longrightarrow} \mathrm{H}^{1}\left(\Delta,\left(\mathbb{D}_{k}^{i+1} / \mathbb{D}_{k}^{i+2}\right)\left(\bar{R}_{\mathcal{U}}, \bar{N}_{\mathcal{U}}\right)\right)
$$

is injective and it is an isomorphism after inverting $p$. It follows that we have isomorphisms $\mathrm{H}^{0}\left(\Delta,\left(\mathbb{D}_{k}^{i} / \mathbb{D}_{k}^{i+2}\right)\left(\bar{R}_{\mathcal{U}}, \bar{N}_{\mathcal{U}}\right)\right) \cong \mathrm{H}^{0}\left(\Delta,\left(\mathbb{D}_{k}^{i+1} / \mathbb{D}_{k}^{i+2}\right)\left(\bar{R}_{\mathcal{U}}, \bar{N}_{\mathcal{U}}\right)\right) \cong \Omega_{w}^{k-2 i-4}(\mathcal{U}) \otimes_{R_{\mathcal{U}}} R_{\mathcal{V}} \otimes_{\mathcal{O}_{K}} \mathcal{O}_{\mathbb{C}_{p}}(i+2)$ where let us recall $\mathcal{V}:=\mathcal{U} \times \times_{\mathcal{X}(w)} \mathcal{X}(p)(w)=\operatorname{Spf}\left(R_{\mathcal{V}}\right)$ and we also have $\left(\widehat{\bar{R}}_{\mathcal{U}}\right)^{\Delta}=R_{\mathcal{V}} \hat{\otimes}_{\mathcal{O}_{K}} \mathcal{O}_{\mathbb{C}_{p}}$. Moreover

$$
\mathrm{H}^{1}\left(\Delta,\left(\mathbb{D}_{k, K}^{i} / \mathbb{D}_{k, K}^{i+2}\right)\left(\bar{R}_{\mathcal{U}}, \bar{N}_{\mathcal{U}}\right)\right) \cong \mathrm{H}^{1}\left(\Delta,\left(\mathbb{D}_{k, K}^{i} / \mathbb{D}_{k, K}^{i+1}\left(\bar{R}_{\mathcal{U}}, \bar{N}_{\mathcal{U}}\right)\right) \cong \omega_{w}^{\dagger, k-2 i}(\mathcal{U}) \otimes_{R_{\mathcal{U}}} R_{\mathcal{V}} \hat{\otimes}_{\mathcal{O}_{K}} \mathbb{C}_{p}(i)\right.
$$

Here we used that the natural map $\mathcal{V}_{K} \longrightarrow \mathcal{U}_{K}$ is (finite) étale and therefore $\Omega_{\mathcal{V}_{K} / K}^{1}=\Omega_{\mathcal{U}_{K} / K}^{1} \otimes_{R_{\mathcal{U}}}$ $R_{\mathcal{V}}$ as $G$-modules. Now we need to compute the $\mathcal{G}$-cohomology of these modules. We have

$$
\mathrm{H}^{0}\left(\mathcal{G},\left(\mathbb{D}_{k}^{i} / \mathbb{D}_{k}^{i+2}\right)\left(\bar{R}_{\mathcal{U}}, \bar{N}_{\mathcal{U}}\right)\right) \cong\left(\mathrm{H}^{0}\left(\Delta,\left(\mathbb{D}_{k}^{i+1} / \mathbb{D}_{k}^{i+2}\right)\left(\bar{R}_{\mathcal{U}}, \bar{N}_{\mathcal{U}}\right)\right)\right)^{G}=\Omega_{w}^{k-2 i-4}(\mathcal{U}) \hat{\otimes}_{\mathcal{O}_{K}} \mathcal{O}_{\mathbb{C}_{p}}(i+2)
$$


Moreover, let us remark for later use that the natural map $\mathrm{H}^{0}\left(\mathcal{G},\left(\mathbb{D}_{k}^{i} / \mathbb{D}_{k}^{i+2}\right)\left(\bar{R}_{\mathcal{U}}, \bar{N}_{\mathcal{U}}\right)\right) \longrightarrow$ $\mathrm{H}^{0}\left(\mathcal{G},\left(\mathbb{D}_{k}^{i} / \mathbb{D}_{k}^{i+1}\right)\left(\bar{R}_{\mathcal{U}}, \bar{N}_{\mathcal{U}}\right)\right)$ is the zero map.

For $\mathrm{H}^{1}$ we shall use the inflation-restriction exact sequence: if $M$ is a continuous $\widehat{\bar{R}}_{\mathcal{U}}$ or indcontinuous $\widehat{\bar{R}}_{\mathcal{U}} \otimes K$-module with continuous semilinear action of $\mathcal{G}$ we have an exact sequence:

$$
0 \longrightarrow \mathrm{H}^{1}\left(G, M^{\Delta}\right) \longrightarrow \mathrm{H}^{1}(\mathcal{G}, M) \longrightarrow \mathrm{H}^{1}(\Delta, M)^{G} \longrightarrow \mathrm{H}^{2}\left(G, M^{\Delta}\right) .
$$

As $G$ has order $p-1$ which is invertible on $M$, and therefore in $M^{\Delta}$ we have $\mathrm{H}^{1}\left(G, M^{\Delta}\right)=$ $\mathrm{H}^{2}\left(G, M^{\Delta}\right)=0$. We obtain that

$$
\mathrm{H}^{1}\left(\mathcal{G},\left(\mathbb{D}_{k, K}^{i} / \mathbb{D}_{k, K}^{i+2}\right)\left(\bar{R}_{\mathcal{U}}, \bar{N}_{\mathcal{U}}\right)\right) \cong\left(\mathrm{H}^{1}\left(\Delta,\left(\mathbb{D}_{k, K}^{i} / \mathbb{D}_{k, K}^{i+2}\right)\left(\bar{R}_{\mathcal{U}}, \bar{N}_{\mathcal{U}}\right)\right)\right)^{G}=\omega^{\dagger, k-2 i}(\mathcal{U}) \hat{\otimes}_{K} \mathbb{C}_{p}(i) .
$$

This proves the case $s=2$. We prove the general case by induction on $s \geq 2$. To shorten notations let us, for the rest of this section denote by by $D^{j}:=\mathbb{D}_{k}^{j}\left(\bar{R}_{\mathcal{U}}, \bar{N}_{\mathcal{U}}\right)$. We have the following commutative diagram with exact rows and columns

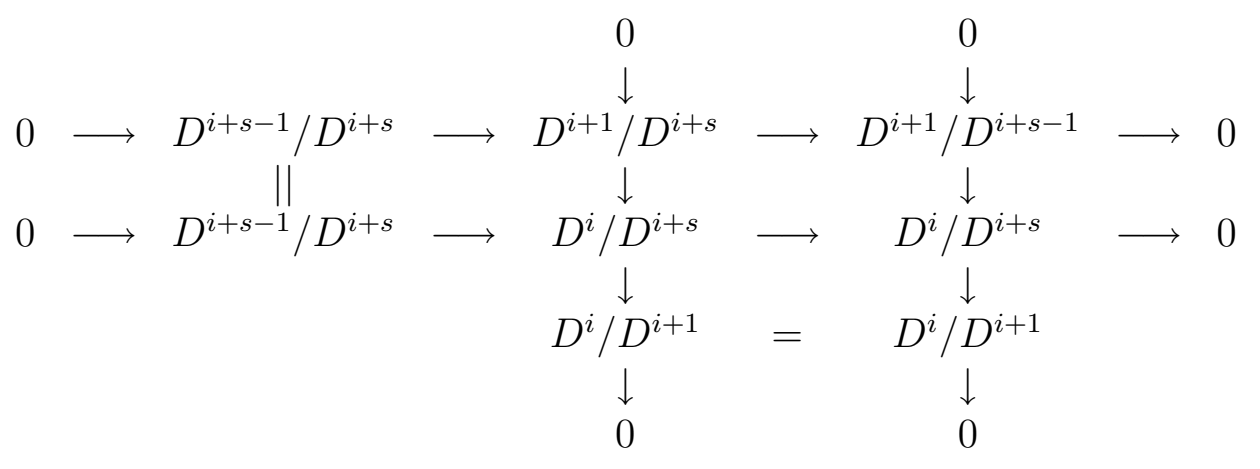

The relevant portion of the associated diagram of cohomology groups is then the following

$$
\begin{array}{ccc}
\mathrm{H}^{0}\left(\Delta, D^{i+1} / D^{i+s-1}\right) & \stackrel{\partial_{s-1}}{\longrightarrow} & \mathrm{H}^{1}\left(\Delta, D^{i+s-1} / D^{i+s}\right) \\
\downarrow \alpha_{s-1} & & \| \\
\mathrm{H}^{0}\left(\Delta, D^{i} / D^{i+s-1}\right) & \stackrel{\partial_{s}}{\longrightarrow} & \mathrm{H}^{1}\left(\Delta, D^{i+s-1} / D^{i+s}\right) \\
\downarrow=0 & & \\
\mathrm{H}^{0}\left(\Delta, D^{i} / D^{i+1}\right) & &
\end{array}
$$

By induction hypothesis the morphism $\partial_{s-1}$ is (an integer)times (an isomorphism) and $\alpha_{s-1}$ is an isomorphism. Therefore $\partial_{s}$ is injective and it becomes an isomorphism after inverting $p$. We obtain

$$
\begin{gathered}
\left.\mathrm{H}^{0}\left(\Delta,\left(\mathbb{D}_{k}^{i} / \mathbb{D}_{k}^{i+s}\right)\left(\bar{R}_{\mathcal{U}}, \bar{N}_{\mathcal{U}}\right)\right) \cong \mathrm{H}^{0}\left(\Delta, \mathbb{D}_{k}^{i+s-1} / \mathbb{D}_{k}^{i+s}\right)\left(\bar{R}_{\mathcal{U}}, \bar{N}_{\mathcal{U}}\right)\right) \cong \\
\cong \Omega_{w}^{k-2 i-2 s+2}(\mathcal{U}) \otimes_{R_{\mathcal{U}}} R_{\mathcal{V}} \hat{\otimes}_{\mathcal{O}_{K}} \mathcal{O}_{\mathbb{C}_{p}}(i+s-1) .
\end{gathered}
$$

Moreover the natural map $\mathrm{H}^{0}\left(\mathcal{G},\left(\mathbb{D}_{k}^{i} / \mathbb{D}_{k}^{i+s}\right)\left(\bar{R}_{\mathcal{U}}, \bar{N}_{\mathcal{U}}\right)\right) \longrightarrow \mathrm{H}^{0}\left(\mathcal{G},\left(\mathbb{D}_{k}^{i} / \mathbb{D}_{k}^{i+s-1}\right)\left(\bar{R}_{\mathcal{U}}, \bar{N}_{\mathcal{U}}\right)\right)$ is the zero map.

We also have

$$
\mathrm{H}^{1}\left(\Delta,\left(\mathbb{D}_{k, K}^{i} / \mathbb{D}_{k, K}^{i+s}\left(\bar{R}_{\mathcal{U}}, \bar{N}_{\mathcal{U}}\right)\right) \cong \mathrm{H}^{1}\left(\Delta,\left(\mathbb{D}_{k, K}^{i} / \mathbb{D}_{k, K}^{i+s-1}\right)\left(\bar{R}_{\mathcal{U}}, \bar{N}_{\mathcal{U}}\right)\right) \cong \omega_{w}^{\dagger, k-2 i}(\mathcal{U}) \otimes_{R_{\mathcal{U}}} R_{\mathcal{V}} \hat{\otimes}_{\mathcal{O}_{K}} \mathbb{C}_{p}(i)\right.
$$

Now we take $G$-invariants reasoning as in the case $s=2$ above and this ends the proof of the proposition. 
Corollary 5.3. We have

i) $R^{0} v_{\mathfrak{X}(w), *}\left(\mathbb{D}_{k}\right)=0$.

ii) $R^{1} v_{\mathfrak{X}(w), *}\left(\mathbb{D}_{k, K} / \mathbb{D}_{k, K}^{s}(1)\right) \cong \omega_{w}^{\dagger, k+2} \hat{\otimes}_{\mathcal{O}_{K}} \mathbb{C}_{p}$, independently of $s \geq 0$.

Proof. Let us first recall that by our conventions $\mathbb{D}_{k}=\mathbb{D}_{k}^{-1}$, therefore ii) follows directly from proposition 5.2. To prove i) let us localize at a small $\log$ affine $\mathcal{U}:=\left(\operatorname{Spf}\left(R_{\mathcal{U}}\right), N_{\mathcal{U}}\right)$ of $\mathcal{X}(w)$ as in the proof of proposition 5.2. We remark that we have an isomorphism as $\widehat{\bar{R}}_{\mathcal{U}}$-modules, $\mathcal{G}$-equivariant

$$
\mathrm{H}^{0}\left(\mathcal{G}, \mathbb{D}_{k}\left(\bar{R}_{\mathcal{U}}, \bar{N}_{\mathcal{U}}\right)\right) \cong \lim _{\leftarrow, s} \mathrm{H}^{0}\left(\mathcal{G},\left(\mathbb{D}_{k} / \mathbb{D}_{k}^{s}\right)\left(\bar{R}_{\mathcal{U}}, \bar{N}_{\mathcal{U}}\right)\right)=0
$$

because the transition maps of the projective system $\left\{\mathrm{H}^{0}\left(\mathcal{G},\left(\mathbb{D}_{k} / \mathbb{D}_{k}^{s}\right)\left(\bar{R}_{\mathcal{U}}, \bar{N}_{\mathcal{U}}\right)\right)\right\}_{s}$ are zero, as can be seen from the proof of proposition 5.2.

Finally we have

Theorem 5.4. Let $k \in \mathcal{W}^{*}(K)$ and $m \geq-1, m \in \mathbb{Z}$. We have

i) $\mathrm{H}^{0}\left(\mathfrak{X}(w), \mathbb{D}_{k, K} / \mathbb{D}_{k, K}^{m}(1)\right)=\mathrm{H}^{0}\left(X(w), \omega_{w}^{\dagger, k-2 m+4}\right) \hat{\otimes}_{K} \mathbb{C}_{p}(m-1)$.

ii) $\mathrm{H}^{1}\left(\mathfrak{X}(w), \mathbb{D}_{k, K} / \mathbb{D}_{k, K}^{m}(1)\right) \cong \mathrm{H}^{0}\left(X(w), \omega_{w}^{\dagger, k+2}\right) \hat{\otimes}_{K} \mathbb{C}_{p}$.

iii) $\mathrm{H}^{i}\left(\mathfrak{X}(w), \mathbb{D}_{k, K} / \mathbb{D}_{k, K}^{m}(1)\right)=0$ for $i \geq 2$.

Proof. i) follows from proposition 5.2 i) and the fact that

$$
\mathrm{H}^{0}\left(\mathfrak{X}(w), \mathbb{D}_{k, K} / \mathbb{D}_{k, K}^{m}(1)\right)=\mathrm{H}^{0}\left(X(w), R^{0} v_{\mathfrak{X}(w), *}\left(\mathbb{D}_{k, K} / \mathbb{D}_{k, K}^{m}(1)\right) .\right.
$$

For ii) let us notice that the Leray spectral sequence degenerates to the following exact sequence

$$
\begin{gathered}
0 \longrightarrow \mathrm{H}^{1}\left(X(w), R^{0} v_{\mathfrak{X}(w), *}\left(\mathbb{D}_{k, K} / \mathbb{D}_{k, K}^{m}(1)\right)\right) \longrightarrow \mathrm{H}^{1}\left(\mathfrak{X}(w), \mathbb{D}_{k, K} / \mathbb{D}_{k, K}^{m}(1)\right) \longrightarrow \\
\longrightarrow \mathrm{H}^{0}\left(X(w), R^{1} v_{\mathfrak{X}(w), *}\left(\mathbb{D}_{k, K} / \mathbb{D}_{k, K}^{m}(1)\right)\right) \longrightarrow \mathrm{H}^{2}\left(X(w), R^{0} v_{\mathfrak{X}(w), *}\left(\mathbb{D}_{k, K} / \mathbb{D}_{k, K}^{m}[1 / p](1)\right)\right) .
\end{gathered}
$$

As $R^{0} v_{\mathfrak{X}(w), *}\left(\mathbb{D}_{k, K} / \mathbb{D}_{k, K}^{m}(1)\right)$ is a coherent sheaf of $\mathcal{O}_{X(w)}$-modules (proposition $5.2 \mathrm{i}$ )) on the affinoid $X(w)$, its cohomology in positive degrees vanishes therefore we obtain the claimed result by applying proposition $5.2 \mathrm{ii})$.

iii) follows in a similar way by using the spectral sequence and proposition 5.2.

\subsection{The $U_{p}$-operator}

In this section we prove the main property of the operator $U_{p}$ with respect to the cohomology of the filtration $\left\{\mathbb{D}_{k}^{h}\right\}_{h \geq-1}$. Henceforth we denote by $\mathbb{D}_{k, w}^{h}$ the continuous sheaf on $\mathfrak{X}(w)$ which was so far denoted by $\mathbb{D}_{k}^{h}$, as in this section we need to keep track of the dependence on $w$.

Theorem 5.5. Let $h \geq 0, k \in \mathcal{W}^{*}(K), 0 \leq w<1 /(p+1)$ and we set $w^{\prime}=p w$.

The operator $U_{p}: \mathrm{H}^{1}\left(\mathfrak{X}\left(w^{\prime}\right), \mathbb{D}_{k, w^{\prime}}^{h}\right) \longrightarrow \mathrm{H}^{1}\left(\mathfrak{X}(w), \mathbb{D}_{k, w}^{h}\right)$ has the property that

$$
U_{p}\left(\mathrm{H}^{1}\left(\mathfrak{X}\left(w^{\prime}\right), \mathbb{D}_{k, w^{\prime}}^{h}\right)\right) \subset p^{h+1}\left(\mathrm{H}^{1}\left(\mathfrak{X}(w), \mathbb{D}_{k, w}^{h}\right)\right) .
$$


Proof. The proof of this theorem is based on an explicit calculation of the action of the isogeny $\pi_{p}^{\vee}$ on the sheaves of distributions on $\mathfrak{X}_{p}^{(1)}(w)$. Let us first recall the universal isogeny of degree $p: \pi_{p}: \mathcal{E} \longrightarrow \mathcal{E} / H$ on $X_{p}^{(1)}(w)$. It induces an exact sequence of groups $0 \longrightarrow H \longrightarrow \mathcal{E} \longrightarrow$ $\mathcal{E} / H \longrightarrow 0$. Dualising we get the exact sequence:

$$
0 \longrightarrow H^{\vee} \longrightarrow(\mathcal{E} / H)^{\vee} \longrightarrow \mathcal{E}^{\vee} \longrightarrow 0
$$

Now we let $\mathcal{U}=(\operatorname{Spf}(R), N)$ be a small affine of $\mathcal{X}(w) \frac{\text { ket }}{K}$ and for every $n \geq 1$ we obtain a commutative diagram with exact rows of $\widehat{\bar{R}}_{\mathcal{U}}$-points

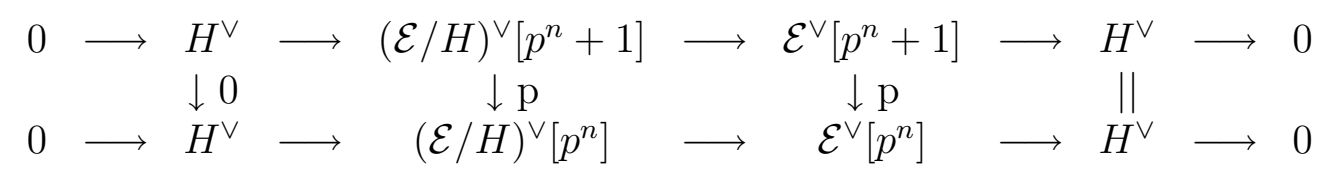

Taking the projective limits with respect to $n$ we obtain an exact sequence of continuous representations of the Kummer étale fundamental group of $\mathcal{U}_{K}$ :

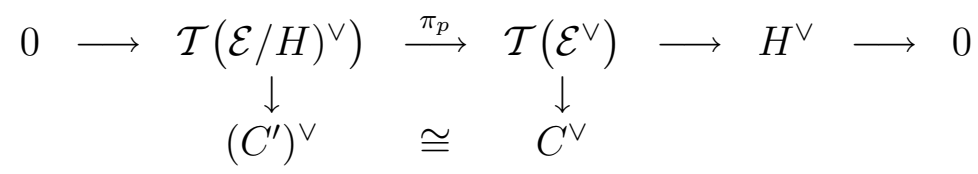

where we have denoted $C \subset \mathcal{E}[p]$ and $C^{\prime} \subset(\mathcal{E} / H)[p]$ the canonical subgroups of $\mathcal{E}$ and $\mathcal{E} / H$,

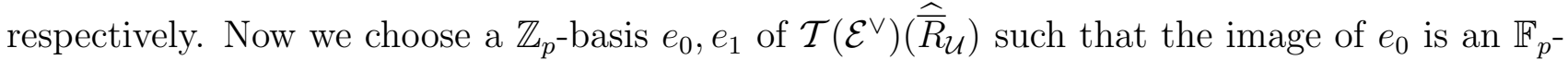
basis of $C^{\vee}$. In this case there is $a \in \mathbb{F}_{p}$ such that $H^{\vee} \cong\left(e_{0} \mathbb{F}_{p} \oplus e_{1} \mathbb{F}_{p}\right) /\left(a e_{1}+e_{0}\right) \mathbb{F}_{p}$. Therefore one can choose a $\mathbb{Z}_{p}$-basis $\left\{e_{0}^{\prime}, e_{1}^{\prime}\right\}$ of $\mathcal{T}\left((\mathcal{E} / H)^{\vee}\right)\left(\widehat{\bar{R}}_{\mathcal{U}}\right)$ such that the image of $e_{0}^{\prime}$ is an $\mathbb{F}_{p \text {-basis }}$ of $\left(C^{\prime}\right)^{\vee}$ and $\pi_{p}\left(e_{0}^{\prime}\right)=[a] e_{1}+e_{0}$ and $\pi_{p}\left(e_{1}^{\prime}\right)=p e_{1}$. Here we denoted by $[a] \in \mathbb{Z}_{p}$ the Teichmüler lift of $a \in \mathbb{F}_{p}$.

Now we'd like to describe the dual map: $\pi_{p}^{\vee}: \mathcal{T}^{\vee}\left(\mathcal{E}^{\vee}\right)(\widehat{\bar{R}}) \longrightarrow \mathcal{T}^{\vee}\left((\mathcal{E} / H)^{\vee}\right)\left(\widehat{\bar{R}}_{\mathcal{U}}\right)$. For this let $\{X, Y\}$ be as at the beginning of section 4.3 , the basis of $\mathcal{T}^{\vee}\left(\mathcal{E}^{\vee}\right)\left(\widehat{\bar{R}}_{\mathcal{U}}\right)$ dual to $\left\{e_{0}, e_{1}\right\}$, i.e., $X\left(e_{0}\right)=1, X\left(e_{1}\right)=0, Y\left(e_{0}\right)=0, Y\left(e_{1}\right)=1$. Let also $A, B$ be the basis of $\left.\mathcal{T}^{\vee}(\mathcal{E} / H)^{\vee}\right)\left(\widehat{\bar{R}}_{\mathcal{U}}\right)$ dual to the basis $\left\{e_{0}^{\prime}, e_{1}^{\prime}\right\}$.

Then $\pi_{p}^{\vee}: \mathcal{T}^{\vee}\left(\mathcal{E}^{\vee}\right)\left(\widehat{\bar{R}}_{\mathcal{U}}\right) \longrightarrow \mathcal{T}^{\vee}\left((\mathcal{E} / H)^{\vee}\right)\left(\widehat{\bar{R}}_{\mathcal{U}}\right)$ is defined by: $\pi_{p}^{\vee}(X)=A, \pi_{p}^{\vee}(Y)=p B+[a] A$. Let us remark that this map is compatible with the map $\pi_{p}^{\vee}: \Omega_{\mathcal{E}}^{1}\left(\widehat{\bar{R}}_{\mathcal{U}}\right) \longrightarrow \Omega_{\mathcal{E} / H}^{1}\left(\widehat{\bar{R}}_{\mathcal{U}}\right)$ given by $\pi_{p}^{\vee}(X)=A$.

Now for $k=(s, j) \in \mathcal{W}^{*}(K)$ and $h \geq-1$ we have the morphism:

$$
\pi_{h}^{k}: \operatorname{fil}_{h}\left(\mathbb{A}_{k}(\mathcal{E})\right) \longrightarrow \operatorname{fil}_{h}\left(\mathbb{A}_{k}(\mathcal{E} / H)\right)
$$

which is given locally

$$
\pi_{h}^{k}: \operatorname{fil}_{h}\left(\mathbb{A}_{k}(\mathcal{E})\right)\left(\widehat{\bar{R}}_{\mathcal{U}}\right)=\left(\sum_{i=0}^{h} \widehat{\bar{R}}_{\mathcal{U}} X^{i} Y^{h-i}\right) X^{k-h} \longrightarrow \operatorname{Fil}^{h}\left(\mathbb{A}_{k}(\mathcal{E} / H)\right)=\left(\sum_{i=0}^{h} \widehat{\bar{R}}_{\mathcal{U}} A^{i} B^{h-i}\right) A^{k-h}
$$

by the formula:

$$
\pi_{h}^{s}\left(\left(\sum_{i=0}^{h} b_{i} X^{i} Y^{h-i}\right) X^{k-h}\right)=\left(\sum_{i=0}^{h} b_{i} A^{i}([a] A+p B)^{h-i} A^{k-h}\right)=\sum_{i=0}^{h} b_{i}([a]+p B / A)^{h-i} A^{k} .
$$


Now we'll work modulo $p^{t}$, i.e., let us recall from section 4.3 that if $k=(s, j)$ and $h \geq-1$ we denote $\mathbb{A}_{k, t}(\mathcal{E}):=\lim _{\rightarrow, n}\left(\operatorname{fil}_{n}\left(\mathbb{A}_{k}(\mathcal{E}) \otimes \mathbb{Z} / p^{t} \mathbb{Z}\right)\right)$ for the transition maps defined at loc. cit., we define by $\mathbb{D}_{k, t}(\mathcal{E}):=\operatorname{Hom}_{\mathcal{O}_{\mathfrak{X}(w)} / p^{t} \mathcal{O}_{\mathfrak{X}(w)}}\left(\mathbb{A}_{k, t}(\mathcal{E}), \mathcal{O}_{\mathfrak{X}(w)} / p^{t} \mathcal{O}_{\mathfrak{X}(w)}\right)$ and $\mathbb{D}_{k, t}^{h}(\mathcal{E}):=\operatorname{Ker}\left(\mathbb{D}_{k, t}(\mathcal{E}) \rightarrow\right.$ $\left.\operatorname{Hom}_{\mathcal{O}_{\mathfrak{X}(w)} / p^{t} \mathcal{O}_{\mathfrak{X}(w)}}\left(\operatorname{fil}_{h}\left(\mathbb{A}_{k, t}(\mathcal{E})\right), \mathcal{O}_{\mathfrak{X}(w)} / p^{t} \mathcal{O}_{\mathfrak{X}(w)}\right)\right)$.

Then (see lemma 4.7)

$\mathbb{D}_{k, t}^{m}(\mathcal{E})\left(\bar{R}_{\mathcal{U}}, \bar{N}_{\mathcal{U}}\right)=\left\{\mu \in \operatorname{Hom}_{\bar{R}_{\mathcal{U}} / p^{t} \bar{R}_{\mathcal{U}}}\left(\mathbb{A}_{k, t}(\mathcal{E})\left(\bar{R}_{\mathcal{U}}, \bar{N}_{\mathcal{U}}\right), \bar{R}_{\mathcal{U}} / p^{t} \bar{R}_{\mathcal{U}}\right)\right.$ such that $\left.\left.\mu\right|_{\mathrm{fil}_{m}\left(\mathbb{A}_{k, t}(\mathcal{E})\right)\left(\bar{R}_{\mathcal{U}}, \bar{N}_{\mathcal{U}}\right)}=0\right\}$

One defines analogously the objects attached to $\mathcal{E} / H$.

Then the morphisms $\pi_{h}^{k}$ modulo $p^{t}$ induce by duality morphisms of sheaves $\rho_{k}^{h}: \mathbb{D}_{k, t}^{h}(\mathcal{E} / H) \longrightarrow$ $\mathbb{D}_{k, t}^{h}(\mathcal{E})$ such that their localization at $\mathcal{U}=\left(\operatorname{Spf}\left(R_{\mathcal{U}}\right), N_{\mathcal{U}}\right)$ is $\rho_{k}^{h}: \mathbb{D}_{k, t}^{h}(\mathcal{E} / H)\left(\bar{R}_{\mathcal{U}}, \bar{N}_{\mathcal{U}}\right) \longrightarrow$ $\mathbb{D}_{k, t}^{h}(\mathcal{E})\left(\bar{R}_{\mathcal{U}}, \bar{N}_{\mathcal{U}}\right)$ given by the formula

$$
\rho_{k}^{h}(\mu)\left(X^{k}\left(\frac{X}{Y}\right)^{n}\right)=\mu\left(A^{k}\left([a]+p \frac{B}{A}\right)^{n}\right)=\sum_{i=0}^{n}\left(\begin{array}{c}
n \\
i
\end{array}\right)[a]^{n-i} p^{i} \mu\left(A^{k}\left(\frac{B}{A}\right)^{i}\right) .
$$

As $\mu \in \mathbb{D}_{k, t}^{h}(\mathcal{E} / H)\left(\bar{R}_{\mathcal{U}}, \bar{N}_{\mathcal{U}}\right)$ we have that $\mu\left(A^{k}\left(\frac{B}{A}\right)^{i}\right)=0$ for $i \leq h$ and so $\rho_{k}^{h}\left(\mathbb{D}_{k, t}^{h}(\mathcal{E} / H)\right) \subset$ $p^{h+1} \mathbb{D}_{k, t}^{h}(\mathcal{E})$ It follows that $\rho_{k}^{h}\left(\mathbb{D}_{k}^{h}(\mathcal{E} / H)\right) \subset p^{h+1} \mathbb{D}_{k}^{h}(\mathcal{E})$ and as the other maps whose composition is $U_{p}$ are $\mathbb{Z}_{p}$-linear, the theorem follows.

\subsection{Slope decomposition}

Let $k \in \mathcal{W}^{*}(K)$ and let us fix $h \in \mathbb{Q}, h \geq 0$. We choose and integer $m$ such that $m-1 \geq h$. Then let us recall that we have a natural $\mathbb{C}_{p}$-linear map commuting with the action of the Hecke operators $T_{\ell}$ for $\ell$ not dividing $N p$ and $U_{p}$ and the Galois group $G_{K}$ :

$$
\mathfrak{R}_{k}: \mathrm{H}^{1}\left(\mathfrak{X}(w), \mathbb{D}_{k, K}(1)\right) \longrightarrow \mathrm{H}^{1}\left(\mathfrak{X}(w), \mathbb{D}_{k, K} / \mathbb{D}_{k, K}^{m}(1)\right) \cong \mathrm{H}^{0}\left(X(w), \omega_{w}^{\dagger, k+2}\right) \hat{\otimes}_{K} \mathbb{C}_{p}
$$

where the last isomorphism is provided by theorem 5.4. Let us recall that we want to prove theorem 5.1 which states that

i) $\mathrm{H}^{1}\left(\mathfrak{X}(w), \mathbb{D}_{k, K}(1)\right)$ has a slope $h$ decomposition for the operator $U_{p}$.

ii) The morphism above induces an isomorphism of Hecke modules:

$$
\mathfrak{R}_{k}^{(h)}: \mathrm{H}^{1}\left(\mathfrak{X}(w), \mathbb{D}_{k, K}(1)\right)^{(h)} \cong \mathrm{H}^{0}\left(X, \omega_{k}^{\dagger, k+2}\right)^{(h)} .
$$

We now define finite slope decompositions and will prove theorem 5.1. We will first recall some definitions and results from $[\mathrm{AS}]$.

Let $Q \in K[T]$ be a polynomial of degree $d$. We define $Q^{*}:=T^{d} Q(1 / T) \in K[T]$. We say that the polynomial $Q$ has slope $h$, for some $h \in \mathbb{Q}$ if the Newton polygon of $Q$ has all slopes smaller or equal to $h$.

Let now $H$ be a $K$ vector space (we do not assume that $H$ has a topological structure) and $u: H \longrightarrow H$ a $K$-linear map. An element $x \in H$ has slope $h$ with respect to $u$ if there is a polynomial $0 \neq Q \in K[T]$ such that:

1) $Q^{*}(u) \cdot x=0$.

2) The slope of $Q$ is $\leq h$.

We set $H^{(h)}$ the subset of $H$ of elements having slope $\leq h$. Then $H^{(h)}$ is a $K$-vector subspace of $H$ and we say that a $K$-vector subspace $M \subset H$ has slope $h$ if $M \subset H^{(h)}$. 
Definition $5.6([\mathrm{AS}])$. A slope $h$ decomposition of $H$ with respect to $u$ is a $K[u]$-module decomposition

$$
H=H_{h} \oplus H_{h}^{*}
$$

such that

a) $H_{h}$ is a finitely generated $K$-vector subspace of $H$ of slope $\leq h$.

b) For every $0 \neq Q \in K[T]$ of slope $\leq h$, the map $Q^{*}(u): H_{h}^{*} \longrightarrow H_{h}^{*}$ is an isomorphism.

Finally we have the following::

Theorem 5.7 ([AS]). 1) If a slope $h$ decomposition of $H$ with respect to $u$ exists, then $H_{h}=H^{(h)}$. 2) If

$$
\text { (*) } A \longrightarrow B \longrightarrow C \longrightarrow D \longrightarrow E
$$

is an exact sequence of $K[u]$-modules and if $A, B, D, E$ have slope $h$ decompositions with respect to $u$ then so does $C$. Moreover, if this happens the exact sequence $(*)$ induces the exact sequence

$$
A^{(h)} \longrightarrow B^{(h)} \longrightarrow C^{(h)} \longrightarrow D^{(h)} \longrightarrow E^{(h)} .
$$

\section{Proof of theorem 5.1}

Let us fix $h \in \mathbb{Q}, h \geq 0, k \in \mathcal{W}^{*}(K)$ and choose $m \in Z$ such that $m \geq h$. We consider the exact sequence of ind-continuous sheaves on $\mathfrak{X}(w)$

$$
0 \longrightarrow \mathbb{D}_{k, K}^{m} \longrightarrow \mathbb{D}_{k, K} \longrightarrow\left(\mathbb{D}_{k, K} / \mathbb{D}_{k, K}^{m}\right) \longrightarrow 0 .
$$

Taking the long exact cohomology sequence we obtain

$$
\begin{gathered}
0 \longrightarrow \mathrm{H}^{0}\left(\mathfrak{X}(w),\left(\mathbb{D}_{k, K} / \mathbb{D}_{k, K}^{m}\right)(1)\right) \longrightarrow \mathrm{H}^{1}\left(\mathfrak{X}(w), \mathbb{D}_{k, K}^{m}(1)\right) \longrightarrow \mathrm{H}^{1}\left(\mathfrak{X}(w), \mathbb{D}_{k, K}(1)\right) \longrightarrow \\
\longrightarrow \mathrm{H}^{1}\left(\mathfrak{X}(w),\left(\mathbb{D}_{k, K} / \mathbb{D}_{k, K}^{m}\right)(1)\right) \longrightarrow \mathrm{H}^{2}\left(\mathfrak{X}(w), \mathbb{D}_{k, K}^{m}(1)\right),
\end{gathered}
$$

We have the operator $U_{p}$-acting on all terms of this sequence compatible with the maps. Using the theorem 5.4 we obtain isomorphisms as Hecke modules

$$
\mathrm{H}^{0}\left(\mathfrak{X}(w),\left(\mathbb{D}_{k, K} / \mathbb{D}_{k, K}^{m}\right)(1)\right) \cong \mathrm{H}^{0}\left(X(w), \omega_{w}^{\dagger, k-2 m+4}\right) \hat{\otimes}_{K} \mathbb{C}_{p}(m-1)
$$

and

$$
\mathrm{H}^{1}\left(\mathfrak{X}(w),\left(\mathbb{D}_{k, K} / \mathbb{D}_{k, K}^{m}\right)(1)\right) \cong \mathrm{H}^{0}\left(X(w), \omega_{w}^{\dagger, k+2}\right) \hat{\otimes}_{K} \mathbb{C}_{p}
$$

By $[\mathrm{Co}]$ as both $\mathrm{H}^{0}\left(\mathfrak{X}(w),\left(\mathbb{D}_{k, K} / \mathbb{D}_{k, K}^{m}\right)(1)\right)$ and $\mathrm{H}^{1}\left(\mathfrak{X}(w),\left(\mathbb{D}_{k, K} / \mathbb{D}_{k, K}^{m}\right)(1)\right)$ are identified as Hecke modules with spaces of overconvergent modular forms, they have slope $h$ decompositions for $U_{p}$.

We now analyse $\mathrm{H}^{i}\left(\mathfrak{X}(w), \mathbb{D}_{k}^{m}(1)\right)$ for $i=1,2$. All we know about the topological structure of these $\mathcal{O}_{\mathbb{C}_{p}}$-modules is that they sit in exact sequences

$$
0 \longrightarrow \lim _{\leftarrow, t}^{(1)} \mathrm{H}^{i-1}\left(\mathfrak{X}(w), \mathbb{D}_{k, t}^{m}(1)\right) \longrightarrow \mathrm{H}^{i}\left(\mathfrak{X}(w), \mathbb{D}_{k}^{m}(1)\right) \longrightarrow \lim _{\leftarrow, t} \mathrm{H}^{i}\left(\mathfrak{X}(w), \mathbb{D}_{k, t}^{m}(1)\right) \longrightarrow 0,
$$

for $i=1,2$. By theorem 5.5 there is a linear operator $U^{\prime}$ on each term of the sequence such that $U_{p}=p^{m+1} U^{\prime}$. Then theorem 5.1 follows from the following general result.

Let us suppose that $\left\{C_{n}\right\}_{n},\left\{B_{n}\right\}_{n}$ are projective systems of abelian groups, $B_{n}$ and $C_{n}$ are $\mathcal{O}_{\mathbb{C}_{p}} / p^{n} \mathcal{O}_{\mathbb{C}_{p}}$-modules for all $n \geq 0$ and $A$ is a $\mathcal{O}_{\mathbb{C}_{p}}$-module such that the following sequence of $\mathcal{O}_{\mathbb{C}_{p}}$-modules is exact

$$
\text { (*) } \quad 0 \longrightarrow \lim _{\leftarrow, n}^{(1)} C_{n} \longrightarrow A \longrightarrow \lim _{\leftarrow, n} B_{n} \longrightarrow 0 .
$$


Moreover let us suppose that there is an $\mathcal{O}_{\mathbb{C}_{p}}$-linear operator $U^{\prime}: A \longrightarrow A$ compatible with the sequence $(*)$, i.e., it induces linear homomorphisms between the projective systems $U^{\prime}:\left\{C_{n}\right\}_{n} \longrightarrow$ $\left\{C_{n}\right\}_{n}$ and $U^{\prime}:\left\{B_{n}\right\}_{n} \longrightarrow\left\{B_{n}\right\}_{n}$. For an integer $m \geq 1$ we denote $U:=p^{m} U^{\prime}$.

Lemma 5.8. For $0 \leq h \leq m-1$ the module $A_{\mathbb{Q}_{p}}=A \otimes_{\mathbb{Z}_{p}} \mathbb{Q}_{p}$ has a slope $h$ decomposition for the operator $U$ and $A_{\mathbb{Q}_{p}}^{(h)}=0$.

Proof. It is enough to show: if $P \in \mathbb{Z}_{p}[T]$ is a monic polynomial such that $P(T)=\prod_{i=1}^{d}\left(T+\alpha_{i}\right)$ with $\alpha_{i} \in \mathcal{O}_{\mathbb{C}_{p}}$ such that $v\left(\alpha_{i}\right) \leq h$ for all $1 \leq i \leq d$ then $P(U)$ is invertible on $A_{\mathbb{Q}_{p}}$.

We choose $\beta_{i} \in \mathcal{O}_{\mathbb{C}_{p}}$ such that $\beta_{i} \alpha_{i}=p^{m}$ for all $1 \leq i \leq d$. Then $\beta_{i} \in p \mathcal{O}_{\mathbb{C}_{p}}$ for all $1 \leq i \leq d$. We denote by $\alpha:=\alpha_{1} \cdot \alpha_{2} \ldots \alpha_{d}$ and

$$
P_{1}(T)=P\left(p^{m} T\right)=\prod_{i=1}^{d}\left(p^{m} T+\alpha_{i}\right)=\alpha \prod_{i=1}^{d}\left(1+\beta_{i} T\right)=\alpha(1+p Q(T))
$$

where $Q(T) \in \mathcal{O}_{\mathbb{C}_{p}}[T]$ is a polynomial. Then $P(U)=P_{1}\left(U^{\prime}\right)=\alpha\left(1+p Q\left(U^{\prime}\right)\right)$.

Claim $1+p Q\left(U^{\prime}\right)$ is invertible on $A$.

Proof of the claim It is enough to prove that $1+p Q\left(U^{\prime}\right)$ is invertible on $\lim _{\leftarrow, n} B_{n}$ and on $\lim _{\leftarrow, n}{ }^{(1)} C_{n}$.

If more generally $\left\{D_{n}\right\}_{n}$ is a projective system of abelian groups with $D_{n}$ a $\mathcal{O}_{\mathbb{C}_{p}} / p^{n} \mathcal{O}_{\mathbb{C}_{p}}$ module for every $n \geq 0$ and we have a linear morphism of projective systems $V:\left\{D_{n}\right\} \longrightarrow$ $\left\{D_{n}\right\}_{n}$ then the operator $1+p Q(V)$ is invertible both on $\lim _{\leftarrow, n} D_{n}$ and $\lim _{\leftarrow, n}{ }^{(1)} D_{n}$, where $Q(T) \in \mathcal{O}_{\mathbb{C}_{p}}[T]$. Let us recall that we have an exact sequence of abelian groups

$$
0 \longrightarrow \lim _{\leftarrow, n} D_{n} \longrightarrow \prod_{n} D_{n} \stackrel{1-\delta}{\longrightarrow} \prod_{n} D_{n} \longrightarrow \lim _{\leftarrow, n}^{(1)} D_{n} \longrightarrow 0
$$

Now let us remark that $1+Q(V)$ is invertible as group homomorphism from $\prod_{n} D_{n} \longrightarrow \prod_{n} D_{n}$, its inverse is $\sum_{i=0}^{\infty}(-1)^{i} p^{i}(Q(V))^{i}$ and it, the inverse that is, commutes with $1-\delta$. This proves the claim and the lemma.

Therefore, coming back to the theorem 5.1, we have an exact sequence of $G_{K}$ and Hecke modules

$$
\begin{aligned}
0 \longrightarrow \mathrm{H}^{0}(\mathfrak{X}(w), & \left.\left(\mathbb{D}_{k, K} / \mathbb{D}_{k, K}^{m}\right)(1)\right) \longrightarrow \mathrm{H}^{1}\left(\mathfrak{X}(w), \mathbb{D}_{k, K}^{m}(1)\right) \longrightarrow \mathrm{H}^{1}\left(\mathfrak{X}(w), \mathbb{D}_{k, K}(1)\right) \longrightarrow \\
\longrightarrow & \mathrm{H}^{1}\left(\mathfrak{X}(w),\left(\mathbb{D}_{k, K} / \mathbb{D}_{k, K}^{m}\right)(1)\right) \longrightarrow \mathrm{H}^{2}\left(\mathfrak{X}(w), \mathbb{D}_{k, K}^{m}(1)\right),
\end{aligned}
$$

where all the terms except possibly $\mathrm{H}^{1}\left(\mathfrak{X}(w), \mathbb{D}_{k}(1)\right)$ have slope $h$ decompositions and the $\mathrm{H}^{2}$ term is zero.

By theorem 5.73$) \mathrm{H}^{1}\left(\mathfrak{X}(w), \mathbb{D}_{k}(1)\right)$ has also a slope $h$ decomposition such that we have an exact sequence

$$
0 \longrightarrow \mathrm{H}^{1}\left(\mathfrak{X}(w), \mathbb{D}_{k, K}^{m}(1)\right)^{(h)}=0 \longrightarrow \mathrm{H}^{1}\left(\mathfrak{X}(w), \mathbb{D}_{k, K}(1)\right)^{(h)} \longrightarrow \mathrm{H}^{0}\left(X(w), \omega_{w}^{\dagger, k+2}\right)^{(h)} \otimes \mathbb{C}_{p} \longrightarrow 0
$$

which proves the claim. 


\section{References}

[AI] F. Andreatta, A. Iovita: Semi-stable sheaves and the comparison isomorphisms for semi-stable formal schemes, A volume in honor of Francesco Baldassarri 60th birthday. Rend. Sem. Mat. Univ. Padova 128 (2012), 131-285

[AIS1] F. Andreatta, A. Iovita, G. Stevens, On overconvergent modular sheaves and modular forms for $\mathbf{G L}_{2 / F}$, Israel Journal of Mathematics 201 (2014), 299-359.

[AIS2] F. Andreatta, A. Iovita, G. Stevens, Overconvergent Eichler-Shimura isomorphisms, to appear in J. Math. Inst. Jussieu.

[AS] A. Ash, G. Stevens, p-Adic slope decomposition, preprint.

[Co] R. Coleman: p-Adic Banach spaces and families of modular forms, Invent. Math. 127 (1997), 417-479.

[Fa] G. Faltings: Hodge-Tate structures and modular forms. Math. Ann. 278 (1987), 133-149.

[Err] F. Andreatta, A. Iovita, Erratum to the article "Global applications to relative $\Phi-\Gamma$ modules, I", Astérisque 330 (2010), p. 543-554.

[HIS] M. Harris, A. Iovita, G. Stevens: A geometric Jacquet-Langlands corespondence.

[Il] L. Illusie, An overview of the work of the work of K. Fujiwara, K. Kato and C. Nakamura on logarithmic étale cohomology, Astérisque 279 (2002), 271-322.

[Ka] K. Kato: Semi-stable reduction and p-adic étale cohomology, Astérisque 223 (1994), 269295.

[Sz] P. Scholze: On torsion in the cohomology of locally symmetric spaces, preprint.

[SzW] P. Scholze, J. Weinstein: Moduli of p-divisible groups, Camb. J. Math. 1 (2013), 145-237. 